\title{
INVESTIGATION OF THE ISING-TYPE MODELS WITHIN CLUSTER APPROACH
}

\author{
R. R. LevitskiI, S. I. Sorokov \\ Institute for Condensed Matter Physics \\ of the Ukrainian Academy of Sciences \\ 1 Svientsitski St., UA-290011 Lviv, Ukraine
}

Received June 14, 1994

\begin{abstract}
The method for calculation of the correlation functions of the Isingtype systems with short-range interaction and with arbitrary value of spin is developed within cluster approximation. For the Ising model (spin $S^{z}= \pm 1$ ) the expressions for pair and ternary correlation functions within two-particle approximation in q-space are obtained for the hypercubic Bravais lattices. In the 1D case the exact expressions for them in the site space is obtained as well. On the basis of the Glauber equation within two-particle cluster approximation the longitudinal dynamical susceptibility $\chi(q, E)$ is found. In the 1D case and in the absence of external field the expression for $\chi(q, E)$ is exact. For the Emery-Blume-Griffiths model $\left(S^{z}=-2,0,2\right)$ within two-particle approximation the pair correlation functions are calculated. The fourparticle cluster approximation is used for calculation of static susceptibility $\chi(\mathbf{q})$ of $\mathrm{KD}_{2} \mathrm{PO}_{4}$ ferroelectrics.
\end{abstract}

\section{Introduction}

The wide class of materials is known which is described on the basis of the pseudospin Hamiltonian. In particular these are magnets with different values of spin $M[1-3]$ (in our notations $S^{z}=-M,-M+2, \ldots, M-2, M$ ), ferroelectrics with hydrogen bonds $[4,5](M=1)$, multicomponent alloys $[6,7]$ $(M+1$ corresponds to the number of components), lattice gas $[8](M+1$ is the number of atom states per site). In the works [9-12] the method of investigation of pseudospin system based on the introducing the reference system (RS) was developed. This approach makes possible to take into account adequately both the short-range and long-range interaction. RS contains besides the low-dimensional short-range $(D=1,2)$ part of the Hamiltonian the terms taking into account the other types of interactions in the molecular field approximation (MFA). The fluctuating corrections to MFA for these types of interactions are taken into account on the basis of expansion of the physical responses (free energy, correlation functions, temperature Green functions) in terms of loop diagrams or in terms of many-tails diagrams. These corrections contain the physical responses of RS. The calculation of the latters is the separate problem of the referense system approach.

In the series of cases for the description of pseudospin system's properties it is enough to take into account the long-range interaction in MFA. Now the reference system problem is to be considered separately. However, 
only a small number of low-dimensional problems may be solved exactly. The exact solutions are obtained mainly in the case of $1 \mathrm{D}$ quantum models or 1D and 2D Ising-type models (ITM) (the Hamiltonian of ITM contains only $S^{z}$ component of the spin). Among approximate methods of investigation the cluster method is sufficiently effective [13-31]. It is necessary to distinguish the cluster expansion method (CEM) and the cluster variation method (CVM). In the case of CEM the cluster expansion is constructed for the free energy [13] whereas in the case of CVM one carries out the cluster expansion of entropy [26]. It can be shown that in the first order of cluster expansion both methods give equivalent results for all responces of pseudospin system. This approximation we shall call the cluster approximation (CA). Let us note that in the first works within the framework of CVM the results were obtained on the basis of combinatoric approach [25] whereas within CEM the results was obtained on the basis of many-particle generalization of MFA [14].

Unfortunately the cluster approach was used mainly for investigation of thermodynamical properties of pseudospin systems. We know only several papers [32-43] where the cluster approach was used for calculation of reference system's correlation functions (CF) (see [32-37]) and Green functions [38-43]. In the works $[32,33]$ the equation for pair CF (Ornstein-Zernike type equation) of ITM was constructed artificially. The dipole-dipole interaction which has the nonanalytical properties at $\mathbf{q} \rightarrow 0$ was taken into account in MFA. The concrete calculations were carried out only in the case $\mathbf{q} \rightarrow 0$. Let us note that only in paraphase the obtained results correspond to cluster approximation. In the work [34] within the frames of CA for ITM the method for calculation of pair CF for RS, when the long-range interaction is absent, was suggested. This method is applicable in the case $T>T_{c}$. Here analytic and numerical investigation of $q$-dependence for CF of some models was performed. 'i he Ising model (IM) (IM corresponds to ITM with $M=1$ ) on square lattice within the frame of two-particle cluster approximation (TPCA) and four-particle cluster approximation (FPCA) was considered. The model of ferroelectrics $\mathrm{KD}_{2} \mathrm{PO}_{4}$ was studied too, In the paper [35] for IM within the frame of cluster expansion method the method which allows to find the correlation function of arbitrary order for RS within cluster approximation was suggested. For IM on hypercubic lattices within TPCA the pair and ternary CF were found. Here it was shown that for 1D system these results coincide with the exact ones. In the ref. [36] it was proved that TPCA for IM yields exact results for all characteristics of the system in the case of tree-like lattice. Moreover in the case of square lattice within CEM the influence of the higher order terms of cluster expansion on thermodynamic characteristics was studied numerically. The pair CF in FPCA was also calculated. In the paper [37] CEM was generalized on the Ising type systems with arbitrary value of spin and detailed numerical investigation of the Blume-Emery-Griffits model was performed.

In the works [38-41] the dynamics of ferroelectrics with hydrogen bonds with accounting of tunneling was investigated within the frames of cluster approach. Here the method of two-time Green's function was used. The equation for them was uncoupled in the spirit of Tyablikov approximation with respect to long-range interaction. At such consideration the shortrange intercluster correlations were not taken into account and intracluster Green's functions appear to be connected only through long-range interaction. This leads to uncorrespondence of expressions for static susceptibility which follows (at $\mathbf{q}=0$ and $\omega_{n}=0$ ) from the expressions for dynamic susceptibility with expressions which are obtained within CA. 
In the works $[42,43]$ a method was developed which within the frames of cluster approach allowed to obtain selfconsistent results for thermodynamic characteristics and temperature Green's functions. Within TPCA the reference Green's functions were calculated for the Heisenberg model [42] and the Ising model in a transverse field [43].

In the present work on the basis of cluster approximation the pseudospin systems which are described by Ising type models, are investigated. In section 2 the statement of CEM for ITM with arbitrary many-particle short-range interaction is presented. In section 3 within TPCA the thermodynamic, correlation functions and relaxational dynamics of IM $(M=1)$ on hypercubic lattice are investigated. In section 4 in the frames of TPCA the Ising type model with pair interaction and arbitrary value of spin is considered. Section 5 is devoted to investigation within TPCA of the Ising type model describing the ferroelectric properties of crystal $\mathrm{KD}_{2} \mathrm{PO}_{4}$.

\section{Statement of the cluster expansion method}

In this section we shall consider the cluster expansion method for the Isingtype model with arbitrary form of the Hamiltonian. In the following sections we shall apply present method to certain models. The Hamiltonian of IM with arbitrary values of spin can be written in the following form $\left(S_{i}^{z}=\right.$ $\left.S_{i}=-M,-M+2, \ldots, M-2, M\right)$

$$
\begin{aligned}
& -\beta^{L} \mathcal{H}={ }^{L} H\left(\left\{h^{(\cdot)}\right\}\right)=H\left(\left\{h^{(\cdot)}\right\}\right)+\frac{1}{2} \sum_{i, j} J_{i j}\left(S_{i}, S_{j}\right), \\
& H\left(\left\{h^{(\cdot)}\right\}\right)=\sum_{i} h_{i}\left(S_{i}\right)+\sum_{(i j)} K_{i j}\left(S_{i}, S_{j}\right)+W(\{S\}), \\
& h_{i}\left(S_{i}\right)=\sum_{\mu=1}^{M} h_{i}^{(\mu)} S_{i}^{\mu} ; K_{i j}\left(S_{i}, S_{j}\right)=\sum_{\mu=1}^{M} \sum_{\nu=1}^{M} K_{i j}^{(\mu, \nu)} S_{i}^{\mu} S_{j}^{\nu}, \\
& J_{i j}\left(S_{i} S_{j}\right)=\sum_{\mu=1}^{M} \sum_{\nu=1}^{M} J_{i j}^{(\mu, i)} S_{i}^{\mu} S_{j}^{\nu} .
\end{aligned}
$$

The one-site part of the Hamiltonian $h_{i}\left(S_{i}\right)$ describes interaction of pseudospins with the fields of different type. The two-site part of the Hamiltonian contains the short-range $K_{i j}\left(S_{i} S_{j}\right)$ and long-range $J_{i j}\left(S_{i} S_{j}\right)$ pair interactions. The three-site, four-site etc. short-range interactions are included in $W(\{S\})$. The pseudospin system with the Hamiltonian $H\left(\left\{h^{(.)}\right\}\right)$ is called the reference system [9-12]. We shall consider the RS which contains only the interaction with the nearest neighbours. In particular, for pair interaction we can write

$$
K_{i j}^{(\mu, \nu)}=K^{(\mu, \nu)} \pi_{i j} ; \pi_{i j}= \begin{cases}1, & i \text { is the nearest neighbour of } j, \\ 0, & \text { in opposite case. }\end{cases}
$$

In the case of the Bravais lattice the indices $i, j$ denote the sites of the lattice. The case of the structure with sublattices we shall consider in section 5. In the present paper the pair long-range interaction is taken into account in the molecular field approximation (MFA). Now the $\mathcal{F}$-function (the logarithm of partition function) of the system with the Hamiltonian (2.1) can be written in the following form 


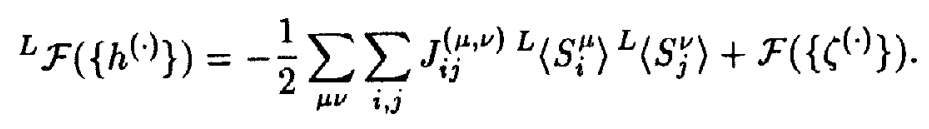

Here $\mathcal{F}\left(\left\{\zeta^{(\cdot)}\right\}\right)$ is $\mathcal{F}$-function of RS with the Hamiltonian containing the long-range molecular field $\lambda_{i}^{(\mu)}$ :

$$
\begin{aligned}
\mathcal{F}\left(\left\{\zeta^{(\cdot)}\right\}\right) & =\ln Z\left(\left\{\zeta^{(\cdot)}\right\}\right) ; Z\left(\left\{\zeta^{(\cdot)}\right\}\right)=S p_{\{s\}} \exp \left[H\left(\left\{\zeta^{(\cdot)}\right\}\right)\right] ; \\
\zeta_{i}^{(\mu)} & =h_{i}^{(\mu)}+\lambda_{i}^{(\mu)} ; \quad \lambda_{i}^{(\mu)}=\sum_{j, \nu} J_{i j}^{(\mu, \nu)} L\left\langle S_{j}^{\nu}\right\rangle .
\end{aligned}
$$

Here the average ${ }^{L}\left\langle S_{i}^{\nu}\right\rangle$ is taken for canonical ensemble and the density matrix is constructed with the Hamiltonian (2.1a). We shall calculate correlation functions (CFs) of RS (cumulant averages of products of operators $\left.S_{i}^{\mu}\right)$ in the following way:

$$
\begin{array}{r}
\mathcal{F}^{(l)}\left(\begin{array}{c}
\mu_{1} \ldots \mu_{l} \\
\mathrm{I}_{1} \ldots i_{l}
\end{array} ;\left\{\zeta^{(\cdot)}\right\}\right)=\left\langle S_{i_{1}}^{\mu_{1}} \ldots S_{i_{l}}^{\mu_{l}}\right\rangle^{c}= \\
=\frac{\delta}{\delta \zeta_{i_{1}}^{\left(\mu_{1}\right)}} \cdots \frac{\delta}{\delta \zeta_{i_{l}}^{\left(\mu_{l}\right)}} \mathcal{F}\left(\left\{\zeta^{(\cdot)}\right\}\right)
\end{array}
$$

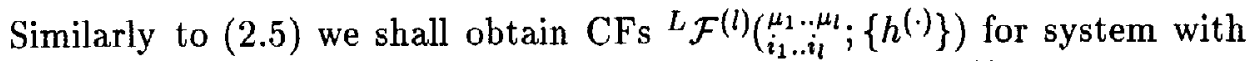
long-range interaction. In this case the $\mathcal{F}$-function ${ }^{L} \mathcal{F}\left(\left\{h^{(\cdot)}\right\}\right)$ is differentiated with respect to fields $h_{j}^{(\mu)}$. In the MFA these CFs can be connected with the CFs of RS. In particular for ${ }^{L}\left\langle S_{i}^{\mu}\right\rangle$ using the relations (2.3), (2.5) we obtain the equality

$$
{ }^{L} \mathcal{F}^{(1)}\left({ }_{i}^{\mu} ;\left\{h^{(\cdot)}\right\}\right)={ }^{L}\left\langle S_{i}^{\mu}\right\rangle=\left\langle S_{i}^{\mu}\right\rangle=\mathcal{F}^{(1)}\left({ }_{i}^{\mu} ;\left\{\zeta^{(\cdot)}\right\}\right)
$$

If we shall differentiate (2.6) and take into account the functional dependence of $\zeta^{(\mu)}$ on $\left\langle S_{i^{\prime}}^{\mu^{\prime}}\right\rangle$, we shall obtain the relation for pair CFs. It has the following matrix form

$$
{ }^{L} \hat{\mathcal{F}}^{(2)}\left(\left\{h^{(\cdot)}\right\}\right)=\hat{\mathcal{F}}^{(2)}\left(\left\{\zeta^{(\cdot)}\right\}\right)\left[1-\hat{J} \hat{\mathcal{F}}^{(2)}\left(\left\{\zeta^{(\cdot)}\right\}\right)\right]^{-1}
$$

Here the matrices $\hat{A}=\left\{A_{i j}^{(\mu, \nu)}\right\}$ are constructed with the help of power-type indices $\mu, \nu$ and indices of sites $i, j$. In the homogeneous field $h_{i}^{(\mu)}=h^{(\mu)}$ the Fourier transformation diagonalizes the relation (2.7) with respect to the indices of the elementary cell.

At first let us decompose lattice into clusters for construction of cluster expansion of $F$-function [36]. The figure 1 demonstrates decomposition of square lattice into clusters.

Let us note that certain choice of lattice decomposition depends on the number of factors. In particular, this choice depends on structure and symmetry of crystal, on form of interaction and cluster expansion order. In the present work we shall use only lattice decomposition into two-particle ("link") and four-particle (tetrahedron) clusters. We shall denote the effective field operator as $R \varphi_{i}\left(S_{i}\right)$. It effects on site $i$ from the side of cluster $R$, 


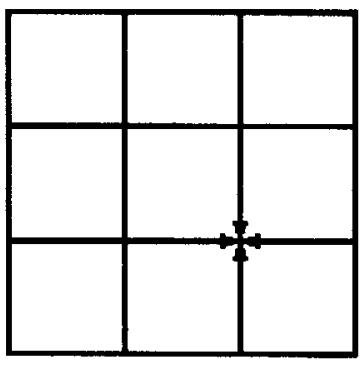

$\mathrm{s}=4, \mathrm{~b}=1, \mathrm{k}=2$

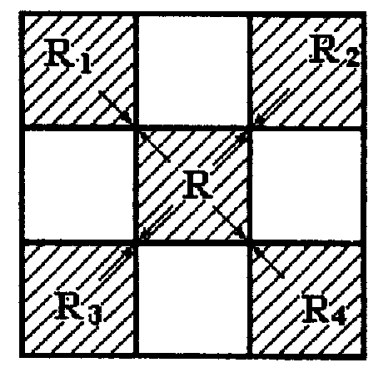

$\mathrm{s}=2, \mathrm{~b}=1, \mathrm{k}=4$

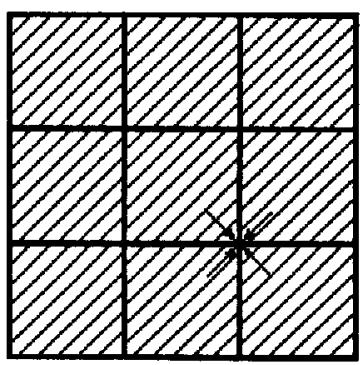

$\mathrm{s}=4, \mathrm{~b}=2, \mathrm{k}=4$

Figure 1: The decomposition of square lattice into clusters ("link", "square") and demonstration of effects of cluster fields on site. Here "s" is the number of clusters containing present site, " $b$ " is the number of clusters containing present link, " $k$ " is the number of sites in cluster.

which contains this site. The set of these clusters we shall mark $\pi_{i}$. Obviously, $s_{i}$ fields effect on an arbitrary site $i$. After decomposition of lattice into clusters we pass from summation over sites to summation over clusters.

$$
\begin{aligned}
& \sum_{i} \sum_{R} R \varphi_{i}\left(S_{i}\right)=\sum_{R} \sum_{f \in R} R \varphi_{f}\left(S_{f}\right) ; W(\{S\})=\sum_{R} W_{R}(\{S\}), \\
& \sum_{(i j)} K_{i j}\left(S_{i} S_{j}\right)=\sum_{R} \sum_{f f^{\prime} \in R} \frac{1}{b_{f f^{\prime}}} K_{f f^{\prime}}\left(S_{f} S_{f^{\prime}}\right) .
\end{aligned}
$$

Here we shall consider the cases when $W_{R}(\{S\})$ contains only spins from the cluster $R$. Taking into account (2.8) after identical transformation the Hamiltonian (2.1) is represented in the following way

$$
H=\sum_{i} H_{i}\left(S_{i}\right)+\sum_{R} U_{R}(\{S\})
$$

where

$$
\begin{aligned}
& H_{i}\left(S_{i}\right)=h_{i}\left(S_{i}\right)+\sum_{R} R \varphi_{f}\left(S_{f}\right), \\
& U_{R}(\{S\})=-\sum_{f \in R} R \varphi_{f}\left(S_{f}\right)+ \\
& +\sum_{f f^{\prime}} \frac{1}{b_{f f^{\prime}}} K_{f f^{\prime}}\left(S_{f} S_{f^{\prime}}\right)+W_{R}(\{S\}) .
\end{aligned}
$$

Then, using the form (2.9) and cluster expansion method (CEM) [13], the $\mathcal{F}$-function can be written in the following form

$$
\mathcal{F}=\sum_{i} F_{i}+\ln \left\langle\exp \left(\sum_{R} U_{R}(\{S\})\right)\right\rangle_{0}=\sum_{i} F_{i}+\sum_{l=1}^{\infty} K_{l}
$$


Here we use the notations

$$
F_{i}=\ln Z_{i}=\ln S p e^{H_{i}} ; \quad \rho_{0}=\prod_{i} \rho_{i}=\prod_{i} \frac{e^{H_{i}}}{Z_{i}}
$$

Functions $K_{1}$ contain sums over sets $\mathbf{I}=\left(R_{1} \cdots R_{l}\right)$, which include noncoinsiding clusters $R_{i}$.

$$
\begin{aligned}
& K_{l}=\sum_{\left(R_{1}, \ldots, R_{l}\right)} K\left(R_{1}, \ldots, R_{l}\right)=\sum_{\{1\}} K(1) \\
& K(\mathrm{l})=\sum_{\nu_{1}=1}^{\infty} \ldots \sum_{\nu_{m}=1}^{\infty} \frac{1}{\nu_{1} !} \ldots \frac{1}{\nu_{m} !}\left\langle U_{R_{1}}^{\nu_{1}} \ldots U_{R_{m}}^{\nu_{m}}\right\rangle_{0} .
\end{aligned}
$$

The cluster functions $K(1)$, as it is well known, are expressed by means of $L(\mathbf{m})$-functions as well as functions $L(\mathbf{l})$ are expressed by means of $K(\mathbf{m})$ in the following way [13]

$$
\begin{array}{r}
K(\mathbf{l})=\sum_{m=1}^{l}(-1)^{l-m} \sum_{\mathbf{m} \subset l} L(\mathbf{m})=L(l)+\sum_{m=1}^{l-1}(-1)^{l-m} \sum_{\mathbf{m} \subset \mathbf{l}} L(\mathbf{m}) \\
L(\mathbf{l})=\sum_{m=1}^{l} \sum_{\mathbf{m} \subset \mathbf{l}} K(\mathbf{l})=K(\mathbf{m})+\sum_{m=1}^{l-1} \sum_{\mathbf{m c l}} K(\mathbf{m})
\end{array}
$$

where $L$-function has the form $(M(1)$-moment function):

$$
\begin{aligned}
& L(1)=\ln M(1)=F(1)-\sum_{i \in \sum_{n=1}^{l} R_{n}} F_{i} \\
& M(1)=\left\langle\exp \left[\sum_{n=1}^{l} U_{R_{n}}\right]\right\rangle_{0} .
\end{aligned}
$$

Here we use the notations 1 for set of clusters, $F(1)$ for $\mathcal{F}$-function, $Z(1)$ for partition function and $H(\mathrm{l})$ for the Hamiltonian:

$$
\begin{aligned}
& F(1)=\ln Z(1)=S p_{\{S\}} \exp \{H(1)\} \\
& H(1)=\sum_{i \in \sum_{n=1}^{l} R_{n}} H_{i}+\sum_{n=1}^{l} U_{R_{n}} .
\end{aligned}
$$

Arbitrary set of clusters 1 of present type forms certain graph (diagram) [36] on the lattice. We shall call graph to be unconnected one if it has parts which do not contain common sites. These parts will be independent statistically, when we shall carry out the averaging with distribution function $\rho_{0}$. Let us separate arbitrary graph $\mathbf{n}$ into two graphs $\mathbf{n}_{1}$ and $\mathbf{n}_{2}$ which have not comon clusters, but which may have common sites.

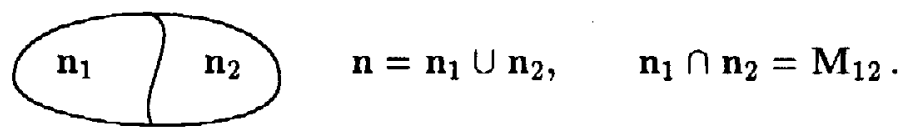


Here $\mathbf{M}_{12}$ is the set of sites, which is common for $\mathbf{n}_{1}$ and $\mathbf{n}_{2}$. Let us represent the sum $\sum_{\mathbf{m} \subset \mathbf{n}}$ in the form $\sum_{\mathbf{m}_{1}} \sum_{\mathbf{m}_{2}}$, where $\mathbf{m}_{1} \subset \mathbf{n}_{1}, \mathbf{m}_{\mathbf{2}} \subset \mathbf{n}_{\mathbf{2}}$. Then we can write (after separation of items with $\mathbf{m}_{1}=0$ and $\mathbf{m}_{2}=0$ )

$$
K(\mathbf{n})=L(\mathbf{n})-L\left(\mathbf{n}_{1}\right)-L\left(\mathbf{n}_{2}\right)-\sum_{m_{1}=1}^{n_{1}} \sum_{m_{2}=1}^{n_{2}} \sum_{\mathbf{m}_{1} \subset \mathbf{n}_{1}} \sum_{\mathbf{m}_{2} \subset \mathbf{n}_{2}} K\left(\mathbf{m}_{1}+\mathbf{m}_{2}\right) \text {. }
$$

The prime near the symbol of sum means that item at $m_{1}=n_{1}, m_{2}=n_{2}$ is omitted. Let the following condition take place

$$
L\left(\mathbf{m}_{1}+\mathbf{m}_{2}\right)=L\left(\mathbf{m}_{1}\right)+L\left(\mathbf{m}_{2}\right), \quad \forall \mathbf{m}_{1} \subset \mathbf{n}_{1}, \mathbf{m}_{2} \subset \mathbf{n}_{2} .
$$

Then we can carry out recurrent procedure for $K\left(\mathbf{m}_{1}+\mathbf{m}_{2}\right)$, which begins with $m_{1}=1, m_{2}=1$. For $\mathbf{m}_{1}=\mathbf{2}_{1}=\mathbf{1}_{1}+\mathbf{1}^{\prime}{ }_{1}, \mathbf{m}_{2}=\mathbf{1}_{1}$ we have

$$
\begin{aligned}
K\left(\mathbf{1}_{1}+\mathbf{1}_{2}\right)= & L\left(\mathbf{1}_{1}+\mathbf{1}_{2}\right)-L\left(\mathbf{1}_{1}\right)-L\left(\mathbf{1}_{2}\right) \equiv 0 \\
K\left(\mathbf{1}_{1}^{\prime}+\mathbf{1}_{2}\right)= & L\left(\mathbf{1}_{1}^{\prime}+\mathbf{1}_{2}\right)-L\left(\mathbf{1}_{1}^{\prime}\right)-L\left(\mathbf{1}_{2}\right) \equiv 0 \\
K\left(\mathbf{2}_{1}+\mathbf{1}_{2}\right)= & L\left(\mathbf{2}_{1}+\mathbf{1}_{2}\right)-L\left(\mathbf{2}_{1}\right)-L\left(\mathbf{1}_{2}\right) \\
& -K\left(\mathbf{1}_{1}+\mathbf{1}_{2}\right)-K\left(\mathbf{1}_{1}^{\prime}+\mathbf{1}_{2}\right) \equiv 0
\end{aligned}
$$

Similarly we can obtain

$$
K\left(\mathbf{m}_{1}+\mathbf{m}_{2}\right) \equiv 0, \quad \forall \mathbf{m}_{1} \subset \mathbf{n}_{1}, \forall \mathbf{m}_{2} \subset \mathbf{n}_{2} .
$$

The condition (2.19) is fulfiled for $n_{1}$ and $n_{2}$ being two unlinked parts of graph $\mathbf{n}$ (when $\mathbf{M}_{12}=0$ ). The condition can be fulfiled also for some linked graphs, if we choose the fields $R \varphi_{i}$ by certain manner (see the following section or paper [36]). Let's note [36] that for set (2.14a) some $L$-functions, which correspond to unlinked diagrams are eliminated by $L$-functions of linked diagrams of certain type. Therefore there is a sum over linked graphs of certain class in (2.14a).

In the present paper we shall use mainly the first approximation for $F$-function and the decomposition of the lattice into $\mathrm{R}$-particle clusters (RPCA). As it follow from (2.11)-(2.16) the $F$-function in RCPA has the form $[36]$

$$
\mathcal{F}\left(\left\{\zeta^{(\cdot)}\right\}\right)=-\sum_{i}\left(s_{i}-1\right) F_{i}\left(\left\{\tilde{\zeta}^{(\cdot)}\right\}\right)+\sum_{R} F_{R}\left(\left\{R^{\prime} \tilde{\zeta}^{(\cdot)}\right\}\right) .
$$

Here the notations for intracluster one-particle $\left(F_{i}\right)$ and R-particle $\left(F_{R}\right)$ $\mathcal{F}$-functions and corresponding partition function $Z_{i}, Z_{R}$ are introduced

$$
\begin{aligned}
& F_{i}\left(\left\{\zeta_{i}^{(\cdot)}\right\}\right)=\ln Z_{i}\left(\left\{\tilde{\zeta}_{i}^{(\cdot)}\right\}\right) ; Z_{i}\left(\left\{\tilde{\zeta}_{i}^{(\cdot)}\right\}\right)=S p_{S_{i}} e^{H_{i}\left(S_{i}\right)} \\
& \left.F_{R}\left(\left\{R^{\prime} \zeta^{(\cdot)}\right\}\right)=\ln Z_{R}\left(\left\{R^{\prime} \tilde{\zeta}^{(\cdot)}\right\}\right) ; Z_{R}\left(\left\{R^{\prime} \tilde{\zeta}^{(\cdot)}\right\}\right)=S p_{\{S\}} e^{H_{R}(S}\right\rangle
\end{aligned}
$$

The one-particle Hamiltonian $H_{i}\left(S_{i}\right)$ and R-particle Hamiltonian $H_{R}(\{S\})$ have the form

$$
\begin{gathered}
H_{i}\left(S_{i}\right)=\sum_{\mu} \tilde{\zeta}_{i}^{(\mu)} S_{i}^{\mu} ; \quad \tilde{\zeta}_{i}^{(\mu)}=\zeta_{i}^{(\mu)}+\sum_{R^{\prime}}{ }_{R} \varphi_{i}^{(\mu)} \\
H_{R}(\{S\})=\sum_{f \in R} H_{j}\left(S_{j}\right)+U_{R}(\{S\})= \\
=\sum_{f \in R} \tilde{\zeta}_{f}\left(S_{f}\right)+\sum_{f f^{\prime} \in R} \frac{1}{b_{f f^{\prime}}} V_{f f^{\prime}}\left(S_{f} S_{f^{\prime}}\right)+W_{R}(\{S\}) ; \\
\tilde{\zeta}_{f}\left(S_{i}\right)=\sum_{\mu} R \tilde{\zeta}_{f}^{(\mu)} S_{f}^{\mu} ; R \tilde{\zeta}_{f}^{(\mu)}=\zeta_{f}^{(\mu)}+\sum_{R^{\prime} \neq R R^{\prime}} \varphi_{f}^{(\mu)} .
\end{gathered}
$$


As we can see from $(2.24 \mathrm{c})$, the fields $R \varphi_{f}^{(\mu)}$ which effect on site $f \subset \mathbf{R}$ from cluster $\mathbf{R}$ are not contained in $\mathbf{R}$-particle Hamiltonian. This is naturally due to the fact that all these interactions are taken into account exactly with the help of terms $V_{f f^{\prime}}$ and $W_{R}$. On the basis of Hamiltonians $H_{i}, H_{R}$ we can introduce intracluster density matrices

$$
\rho_{i}\left(S_{i}\right)=\frac{e^{H_{i}\left(S_{i}\right)}}{Z_{i}} ; \rho_{R}(\{S\})=\frac{e^{H_{R}(\{S\})}}{Z_{R}},
$$

and intracluster CFs

$$
\begin{aligned}
& \left\langle\left(S_{i}\right)^{l_{1}^{i}}\left(S_{i}^{2}\right)^{l_{2}^{i}} \ldots\left(S_{i}^{M}\right)^{l_{M}^{i}}\right\rangle_{\rho_{i}}^{c}, \\
& \left\langle\left(S_{f_{1}}\right)^{K_{1}^{f_{1}}} \ldots\left(S_{f_{1}}^{M}\right)^{K_{M}^{f_{1}}} \ldots\left(S_{f_{R}}\right)^{K_{1}^{f_{R}}} \ldots\left(S_{f_{R}}^{M}\right)^{K_{M}^{f_{R}}}\right\rangle_{\rho_{R}}^{c} \text {. }
\end{aligned}
$$

We shall calculate CFs (2.26) on the basis of $F$-functions (2.23) and relations of (2.4)-type. Evidently, they do not equal zero only if the site indices ( $i$ or $f$ ) belong to present cluster (i or $\mathbf{R}$ ).

We shall find the system of equations for the fields $R \varphi_{i}^{(\mu)}$ from condition of stationarity of $\mathcal{F}$-function with respect to these fields. In the present paper we shall consider only RPCA. As it will be written in the following sections the system of $N s_{i} M$ equations in RCPA has the form

$$
\left\langle S_{i}^{\mu}\right\rangle_{\rho_{i}}=\left\langle S_{i}^{\mu}\right\rangle_{\rho_{R}}
$$

Here the average is defined with respect to density matrices (2.25). The system (2.27) is equivalent to the system of $N s_{i}(M+1)$ equations

$$
\rho_{i}\left(S_{i}\right)=S p_{\mathbf{R}-\mathbf{i}} \rho_{R}(\{S\}),
$$

which coincides with the exact relations between one particle and $\mathrm{R}$-particle density matrices. Among $N s_{i}(M+1)$ equations (2.28) only $N s_{i} M$ ones are independent because $N s_{i}$ conditions $S p_{i} S p_{\mathbf{R}-\mathbf{i}} \rho_{r}(\{S\})=1$ are fulfiled identically. In the homogeneous field $\left(h_{i}=h, \zeta_{i}=\zeta, R \varphi_{i}^{(\mu)}=\varphi^{(\mu)}\right)(2.27)$ gives $M$ equations for $M$ unknown fields $\varphi^{(\mu)}$.

\section{Thermodynamic and dynamic properties of the Ising model. Two-particle cluster approxi- mation}

\subsection{The main relations. Thermodynamic properties}

In the present section we shall consider the Ising-type model at $M=1, S_{i}=$ \pm 1 (Ising model-IM) in the case of hypercubic lattice. Now the Hamiltonian (2.1) contains only linear and bilinear forms of pseudospin operators and we can write it in the following way

$$
H(\{\zeta\})=\sum_{i} \zeta_{i} S_{i}+\sum_{(i j)} K_{i j} S_{i} S_{j} ; \quad K_{i j}=\beta K \pi_{i j}
$$

In TPCA the cluster contains only two sites $\left(\mathbf{R}_{1 r}=(1, r)\right)$ and we can speak about effect on the site $i$ from it's neighbour $r$. This fact leads to the change 
of notations for the cluster fields: $R_{R} \varphi_{1} \rightarrow{ }_{r} \varphi_{1}$. Thus, taking into account $(2.22)-(2.24)$, we obtain $\left(s_{i}=z, b_{f f^{\prime}}=1\right)[35]$

$$
\mathcal{F}(\{\zeta\})=(1-z) \sum_{1} F_{1}\left(\tilde{\zeta}_{1}\right)+\frac{1}{2} \sum_{1, r} F_{1 r}\left(\left.{ }_{r} \tilde{\zeta}_{1}\right|_{1} \tilde{\zeta}_{r}\right)
$$

The one-particle and two-particle cluster Hamiltonians have the form

$$
\begin{gathered}
H_{1}\left(S_{1}\right)=\tilde{\zeta}_{1} S_{1} ; \tilde{\zeta}_{1}=\zeta_{1}+\sum_{r^{\prime} \in \pi_{1}} r^{\prime} \varphi_{1}, \\
H_{12}\left(S_{1}, S_{2}\right)=2 \tilde{\zeta}_{1} S_{1}+_{1} \tilde{\zeta}_{2} S_{2}+K S_{1} S_{2}, \\
{ }_{2} \tilde{\zeta}_{1}=\zeta_{1}+\sum_{r^{\prime} \in \pi_{1} ; r^{\prime} \neq 2} r^{\prime} \varphi_{1} ;{ }_{1} \tilde{\zeta}_{2}=\zeta_{2}+\sum_{r^{\prime} \in \pi_{2} ; r^{\prime} \neq 1} r^{\prime} \varphi_{2} .
\end{gathered}
$$

Thus, the following expressions are obtained for the intracluster $\mathcal{F}$-functions:

$$
\begin{gathered}
F_{1}\left(\tilde{\zeta}_{1}\right)=F_{1}^{(0)}=\ln \left\{2 \cosh \tilde{\zeta}_{1}\right\} \\
F_{1 r}=F_{1 r}^{(00)}= \\
=\ln \left\{2 e^{\beta K}\left[\cosh \left({ }_{r} \tilde{\zeta}_{1}+{ }_{1} \tilde{\zeta}_{r}\right)+e^{-\beta K} \cosh \left({ }_{r} \tilde{\zeta}_{1}-{ }_{1} \tilde{\zeta}_{r}\right)\right]\right\} .
\end{gathered}
$$

If we take into account the long range interaction in MFA we must renormalize the field $\zeta_{i}: \zeta_{i}=h_{i}+\lambda_{i}=h_{i}+\sum_{j} J_{i j}\left\langle S_{j}\right\rangle$. Here $h_{i}$ is an external field, $\lambda_{i}$ a long-range-acting molecular field.

In the present section we shall use the following notations

$$
\begin{aligned}
& \mathcal{F}_{i_{1} \ldots i_{R}}^{(l)}(\{\zeta\})=\left\langle S_{i_{1}} \ldots S_{i_{R}}\right\rangle^{c}=\frac{\delta}{\delta \zeta_{i_{1}}} \cdots \frac{\delta}{\delta \zeta_{i_{l}}} \mathcal{F}(\{\zeta\}), \\
& \tilde{\zeta}_{i_{j}}^{(1)}=\delta_{i j}+\sum_{r \in \pi_{i}} r \varphi_{i_{j}}^{(1)}=\frac{\delta}{\delta \zeta_{j}} \tilde{\zeta}_{i}, \\
& \tilde{\zeta}_{i_{1} i_{2} \ldots i_{l}}^{(l-1)}=\frac{\delta}{\delta \zeta_{i_{2}}} \cdots \frac{\delta}{\delta \zeta_{i_{l}}} \tilde{\zeta}_{i_{1}}=\sum_{r \in \pi_{i_{1}}} r \varphi_{i_{1} \ldots i_{l}}^{(l-1)} ; l \geq 3, \\
& { }_{r} \varphi_{i_{1} \ldots i_{l}}^{(l-1)}=\frac{\delta}{\delta \zeta_{i_{2}}} \cdots \frac{\delta}{\delta \zeta_{i_{l}}} r \varphi_{i_{1}}, \\
& F_{i_{1}}^{(l)}\left(\tilde{\zeta}_{1}\right)=\frac{\partial_{1}^{l}}{\partial\left(\tilde{\zeta}_{1}\right)^{l}} F_{1}^{(0)}\left(\tilde{\zeta}_{1}\right)=\left\langle S^{l}\right\rangle_{\rho_{1}}^{c}, \\
& F_{12}^{\left(l_{1} l_{2}\right)}\left(\left.{ }_{2} \tilde{\zeta}_{1}\right|_{1} \tilde{\zeta}_{2}\right)=\frac{\partial^{l_{1}}}{\partial\left(_{2} \tilde{\zeta}_{1}\right)^{l_{1}}} \frac{\partial^{l_{2}}}{\partial\left(\tilde{\zeta}_{2} \tilde{\zeta}^{l_{2}}\right.} F_{12}^{(00)}=\left\langle S_{1}^{l_{1}} S_{1}^{l_{2}}\right\rangle_{\rho_{12}}^{c} \cdot
\end{aligned}
$$

Now taking into account (2.5) we easily obtain from (3.2)

$$
\left\langle S_{1}\right\rangle=\mathcal{F}_{1}^{(1)}(\{\zeta\})=\frac{\partial}{\partial \zeta_{1}} \mathcal{F}(\{\zeta\})+\sum_{1^{\prime}, \tau} \frac{\partial}{\partial_{r} \varphi_{1^{\prime}}} \mathcal{F}(\{\zeta\})_{r} \varphi_{1^{\prime} 1}^{(1)}
$$

Here 


$$
\begin{aligned}
& \frac{\partial}{\partial \zeta_{1}} \mathcal{F}(\{\zeta\})=(1-z) F_{1}^{(1)}+\sum_{r^{\prime}} F_{1 r^{\prime}}^{10}, \\
& \frac{\partial}{\partial_{r} \varphi_{1^{\prime}}} \mathcal{F}(\{\zeta\})=(1-z) F_{1^{\prime}}^{(1)}+\sum_{r^{\prime}} F_{1^{\prime} \tau^{\prime}}^{10}-F_{1^{\prime} \tau}^{10} .
\end{aligned}
$$

Proceeding from stationarity condition for $\mathcal{F}(\{\zeta\})$ with respect to ${ }_{r} \varphi_{1}$ $\left(\frac{\partial}{\partial_{r} \varphi_{1}} \mathcal{F}(\{\zeta\})=0\right)$ on the basis of (3.6) and (3.7) we find the following relations

$$
\begin{gathered}
m_{1}=\left\langle S_{1}\right\rangle=\mathcal{F}_{1}^{(1)}(\{\zeta\})=F_{1}^{(1)}\left(\left\{\tilde{\zeta}_{1}\right\}\right), \\
\left\langle S_{1}\right\rangle_{\rho_{1}}=F_{1}^{(1)}\left(\left\{\tilde{\zeta}_{1}\right\}\right)=F_{1 r}^{(10)}\left(\left.{ }_{r} \tilde{\zeta}_{1}\right|_{1} \tilde{\zeta}_{r}\right)=\left\langle S_{1}\right\rangle_{\rho_{1 r}} .
\end{gathered}
$$

If the long-range interaction is absent (3.8b) gives the system of $N z$ equations for $N z$ unknown variational fields ${ }_{r} \varphi_{i}(\{\zeta\}, K)$ while $(3.8 \mathrm{a})$ gives the expression for the average value $\left\langle S_{1}\right\rangle$. The expression $(3.8 \mathrm{~b})$ is equivalent to the relation between one-particle $\rho_{1}$ and two-particle $\rho_{1 r}$ density matrices:

$$
\rho_{1}\left(S_{1}\right)=S p_{S_{r}} \rho_{1 r}\left(S_{1}, S_{r}\right) \text {. }
$$

This equivalence can be proved if one takes into account that among $2 N z$ equations (3.9) only $N z$ equations are independent because $N Z$ conditions $S p_{S_{1} S_{r}} \rho_{1 r}\left(S_{1}, S_{r}\right)=S p_{S_{1}} \rho_{1}\left(S_{1}\right)=1$ are accomplished. It follows from (3.9) that

$$
\left.F_{1}^{(l)}\left(\tilde{\zeta}_{1}\right)=\left\langle\left(S_{1}\right)^{l}\right\rangle_{\rho_{1}}^{c}=\left\langle\left(S_{1}\right)^{l}\right\rangle_{\rho_{12}}^{c}=\left.F_{1 r}^{(l, 0)}{ }_{r} \tilde{\zeta}_{1}\right|_{1} \tilde{\zeta}_{r}\right)
$$

The equation (3.9) can be written in the other form which is useful for diagram analysis

$$
M_{(1 r)}=S p_{S_{1} S_{r}}\left(\rho_{1} \rho_{r} e^{U_{1 r}}\right)=S p_{S_{r}} \rho_{r} e^{U_{1 r}} .
$$

Let us consider in expansion for $\mathcal{F}$-function certain type of graphs which contains links that connect the site 1 with another part of the present graph [36]. In particular, this type of diagrams includes tree-graph, that is graph of links without loops.
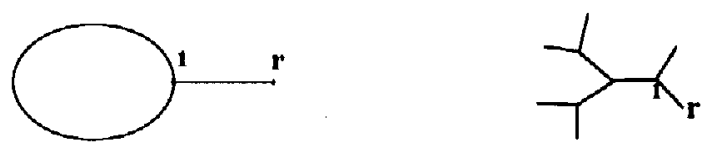

Let us denote by $\nu$ the set of all sites of graph $\mathbf{n}$. Then for the moment function of graph (3.12) taking into account (3.11) we can write

$$
M(\mathbf{n}+(1 r))=S p_{\left\{S_{j}\right\}} \prod_{j \in \nu} \rho_{j} \exp \left(\sum_{\mathbf{R} \in \mathbf{n}} U_{R}\right) S p \rho_{r} e^{U_{1 r}}=M(\mathbf{n}) M(1 r) .
$$

Since $M$-function for the graph (3.12) is factorized, the corresponding $L$ function has the additive form (2.19). This fact means that for the graph of (3.12)-type the $K$ function is equal to zero. It is shown in [36] that the differentiation $\frac{\partial}{\partial_{r} \varphi_{1}}$ of $K$-function for tree type graphs also gives zero. But for $K$-function of arbitrary graph this condition is not fulfilled (when 
the index $i$ in operator $\frac{\partial}{\partial \varphi_{i}}$ coincides with $r$ in (3.12) and $\mathbf{n}$ does not contain links with free tails). Thus if in the expansion of $\mathcal{F}$-function we shall consider only tree-graph and function ${ }_{r} \varphi_{i}(\{\zeta\}, K)$ will satisfy the system (3.11) (or identical system (3.8b)) then the stationarity of $\mathcal{F}$-function with respect to fields will have been accomplished. In this case the expressions for $\mathcal{F}$-function and $\left\langle S_{1}\right\rangle$ coincide with expressions $(3.2)$ and $(3.8 \mathrm{a})$ which are obtained in TPCA. It follows that for the lattice of the tree type, e.g. for $1 D$ chain, the TPCA gives an exact results for all quantities of the IM with Hamiltonian (3.1) $[35,36]$.

Now we pay attention to thermodynamical properties of IM in TPCA taking into account the long-range interaction in MFA. In the case of homogeneous field extracting $\beta$ we can write

$$
\begin{aligned}
& r \varphi_{i}=\beta \varphi ; \sum_{r}{ }_{r} \varphi_{i}=z \beta \varphi ; \zeta_{i}=\beta \zeta=\beta h+\beta J_{0} m \\
& \tilde{\zeta}_{i}=\tilde{\zeta}=\beta(\zeta+z \varphi) ;{ }_{r} \tilde{\zeta}_{i}=\beta \tilde{\tilde{\zeta}}=\beta[\zeta+(z-1) \varphi]
\end{aligned}
$$

where $J_{0}=J(\mathbf{q} \rightarrow \mathbf{0})$. From (3.2), (2.23) and (3.14) for the free energy per site $f(h, K)$ we obtain the following result

$$
\begin{gathered}
\beta^{L} f(h, K, J)=\frac{1}{2} J_{0} m^{2}+\beta f(\zeta, K) \\
-\beta f(\zeta, K)=\frac{1}{N} \mathcal{F}(\zeta)=(1-z) \ln Z_{1}(\tilde{\zeta})+\frac{z}{2} \ln Z_{12}(\tilde{\widetilde{\zeta}})
\end{gathered}
$$

Here the following notation is used

$$
\begin{aligned}
& Z_{1}(\tilde{\zeta})=2 \cosh (\beta \tilde{\zeta}) ; \quad a=e^{-2 \beta K} \\
& Z_{12}(\tilde{\tilde{\zeta}})=2 e^{\beta K}[\cosh (2 \beta \tilde{\tilde{\zeta}})+a] .
\end{aligned}
$$

The system of equations (3.8) for $m$ and $\varphi$ has the form

$$
\begin{aligned}
& m=\tanh [\beta(\zeta+z \varphi)] ; z \varphi=-\zeta+(2 \beta)^{-1} \ln \frac{1+m}{1-m} \\
& \tanh [\beta(\zeta+z \varphi)]=\sinh (2 \beta \tilde{\tilde{\zeta}})[\cosh (2 \beta \tilde{\tilde{\zeta}})+a]^{-1} .
\end{aligned}
$$

In the case $J_{0}=0$ the system (3.17) leads to single equation for $\varphi(\beta h, \beta K)$ (or for $m(\beta h, \beta K)$ ) if we exclude $m$ (or $\varphi$ ) from (3.17a). In the 1D case $\left(J_{0}=0, z=2\right)$ the equation $(3.17 \mathrm{~b})$ for $\varphi$ can be solved exactly, and for $m$ and $f$ from (3.15) and (3.17a) we get the generally known exact results $[44,45]$

$$
\begin{aligned}
& -\beta f=K+\ln [\cosh \beta K+r], \\
& m=\frac{\sinh (\beta h)}{r} ; \quad r=\sqrt{a^{2}+\sinh ^{2}(\beta h)} .
\end{aligned}
$$

The equation (3.17b) can be used to obtain expressions for $\frac{\partial \varphi}{\partial \zeta}$, $\frac{\partial \varphi}{\partial h}$. It makes possible to find the pair correlation function $\left(\left\langle S_{i} S_{j}\right\rangle^{c}\right)_{\mathbf{q}}=F_{\mathrm{q}}^{(2)}$ at $\mathbf{q}=0$ and longitudinal isothermic susceptibility $\chi^{z z}(\mathbf{q}=0)=\chi_{0}^{z z}$ for IM:

$$
{ }^{L} \mathcal{F}_{\mathbf{q}=0}^{(2)}=\frac{\delta m}{\delta h}=\left[\left(\mathcal{F}_{0}^{(2)}\right)^{-1}-\beta J_{0}\right]^{-1} ; \chi_{0}^{z z}=\beta \frac{d^{2}}{v_{a}}{ }^{L} \mathcal{F}_{\mathbf{q}=0}^{2}
$$


Here $d$ is the dipole electric (or magnetic) moment per one cell, $v_{a}$ is the volume of elementary cell,

$$
\left(\mathcal{F}_{0}^{(2)}\right)^{-1}=\frac{z}{2} \frac{[a+\cosh (2 \beta \tilde{\tilde{\zeta}})]^{2}}{1+a \cosh (2 \beta \tilde{\tilde{\zeta}})}-\frac{z-1}{1-m^{2}} .
$$

In the 1D case from (3.20) using solution for $\varphi$ one obtains the expression

$$
\left(\mathcal{F}_{0}^{(2)}\right)^{-1}=r^{3}\left[a^{2} \cosh (\beta h)\right]^{-1} .
$$

On the basis of (3.15) one can easy obtain other thermodynamic quantities of IM in TCPA, in particular, entropy and specific heat. In the case $h=0$ the equation (3.17) for $\varphi$ (or $m$ ) always has the solution $\varphi=0(m=0)$ and at $T<T_{c}$ (below the phase transition temperature) these equations have also non-trivial solution $\varphi \neq 0(m \neq 0)$. As it was shown in [36], in TCPA for IM at $K>0$ the phase transition (PT) of the second order takes place. The equation for $T_{c}$ is found from divergence condition for ${ }^{L} \mathcal{F}_{0}^{(2)}$

$$
z \exp \left[-2 \beta_{c} K\right]-z+2=2 \beta_{c} J_{0} \text {. }
$$

At $J_{0}=0$ from $(3.22)$ one obtains well known result for $T_{c}$ of IM in TCPA $[44,45]$

$$
k_{B} T_{c}=2 K\left[\ln \frac{z}{z-2}\right]^{-1} .
$$

In the $1 \mathrm{D}$ case $(z=2)$ from $(3.23)$ we find that $T_{c}=0$. Thus for $1 \mathrm{D}$ IM the $\mathrm{PT}$ is absent at temperatures above zero.

\subsection{The correlation furctions}

In the present section we are going to consider briefly the suggested method for calculation of the correlation functions of IM in the cluster approach. We are going to find the explicit expressions for the pair and ternary CFs in the wave vector space. The results and the method of calculation of CFs in the site space in 1D case are presented in the work [35].

On the basis of relations (2.5) and (3.8a) CFs can be expressed with the. help of derivatives of fields $\tilde{\zeta}_{i}$ (see $(3.5 \mathrm{~b})$ ) with respect to $\zeta_{j}$. We present the general expressions for pair, ternary and four-spin CFs in the wave vector space [35]

$$
\begin{gathered}
\mathcal{F}^{(2)}\left(\mathbf{q}_{1}\right)=F^{(2)} \tilde{\zeta}^{(1)}\left(\mathbf{q}_{1}\right) \\
\mathcal{F}^{(3)}\left(\mathbf{q}_{1} \mathbf{q}_{2}\right)=F^{(3)} \tilde{\zeta}^{(1)}\left(\mathbf{q}_{1}\right) \tilde{\zeta}^{(1)}\left(\mathbf{q}_{2}\right)+F^{(2)} \tilde{\zeta}^{(2)}\left(\mathbf{q}_{1} \mathbf{q}_{2}\right) \\
\mathcal{F}^{(4)}\left(\mathbf{q}_{1} \mathbf{q}_{2} \mathbf{q}_{3}\right)=F^{(4)} \tilde{\zeta}^{(1)}\left(\mathbf{q}_{1}\right) \tilde{\zeta}^{(1)}\left(\mathbf{q}_{2}\right) \tilde{\zeta}^{(1)}\left(\mathbf{q}_{3}\right)+ \\
+F^{(2)} \tilde{\zeta}^{(3)}\left(\mathbf{q}_{1} \mathbf{q}_{2} \mathbf{q}_{3}\right)+F^{(3)}\left[\tilde{\zeta}^{(1)}\left(\mathbf{q}_{1}\right) \tilde{\zeta}^{(2)}\left(\mathbf{q}_{2} \mathbf{q}_{3}\right)+\right. \\
\left.+\tilde{\zeta}^{(1)}\left(\mathbf{q}_{2}\right) \tilde{\zeta}^{(2)}\left(\mathbf{q}_{1} \mathbf{q}_{3}\right)+\tilde{\zeta}^{(1)}\left(\mathbf{q}_{3}\right) \tilde{\zeta}^{(2)}\left(\mathbf{q}_{1} \mathbf{q}_{2}\right)\right] \\
F^{(2)}=1-m^{2} ; F^{(3)}=-2 m F^{(2)} ; F^{(4)}=-2 F^{(2)}\left(1-3 m^{2}\right) .
\end{gathered}
$$

Thus it is necessary to construct the equations for $\tilde{\zeta}^{(1)}(\mathbf{q}), \tilde{\zeta}^{(2)}\left(\mathbf{q}_{1}, \mathbf{q}_{2}\right)$ and $\tilde{\zeta}^{(3)}\left(\mathbf{q}_{1}, \mathbf{q}_{2}, \mathbf{q}_{3}\right)$ (on the basis of $(3.8 \mathrm{~b})$ ) for the computation of $\mathcal{F}^{(2)}(\mathbf{q})$, $\mathcal{F}^{(3)}\left(q_{1}, q_{2}\right)$ and $\mathcal{F}^{(4)}\left(q_{1}, q_{2}, q_{3}\right)$. 
Now we are going to consider the equation for $\tilde{\zeta}^{(\mathbf{1})}(\mathbf{q})$. Differentiating (3.8b) with respect to $\zeta_{2}$ one obtains

$$
\begin{aligned}
& F_{1}^{(2)} \tilde{\zeta}_{12}^{(1)}=F_{1 r}^{(20)}{ }_{r} \tilde{\zeta}_{12}^{(1)}+F_{1 r}^{(11)}{ }_{1} \tilde{\zeta}_{r 2}^{(1)} \\
& { }_{r} \tilde{\zeta}_{12}^{(1)}=\tilde{\zeta}_{12}^{(1)}-{ }_{r} \varphi_{12}^{(1)} ;{ }_{1} \tilde{\zeta}_{r 2}^{(1)}=\tilde{\zeta}_{r 2}^{(1)}-{ }_{1} \varphi_{r 2}^{(1)} .
\end{aligned}
$$

Taking into account (3.24b) and (3.10) the equation (3.24a) and the same equation with replaced indices $1 \leftrightarrow r$ constitute the system of equations for the fields ${ }_{r} \varphi_{12}^{(1)}$ and ${ }_{1} \varphi_{r 2}^{(1)}$. This system can be written in the form

$$
\left(\begin{array}{cc}
1 & f_{1 r} \\
f_{r 1} & 1
\end{array}\right)\left(\begin{array}{c}
r \varphi_{12}^{(1)} \\
{ }_{1} \varphi_{r 2}^{(1)}
\end{array}\right)=\left(\begin{array}{c}
f_{1 r} \tilde{\zeta}_{r 2}^{(1)} \\
f_{r 1} \bar{\zeta}_{12}^{(1)}
\end{array}\right) ; f_{1 r}=\frac{F_{1 r}^{(11)}}{F_{1 r}^{(20)}}
$$

From (3.25) one can easy express ${ }_{r} \varphi_{i j}^{(1)}$ in terms of $\tilde{\zeta}_{i j}^{(1)}$. Taking into consideration the relationship (3.5b) between $\zeta_{i j}^{(1)}$ and $\sum_{r} \varphi_{i j}^{(1)}$ one can obtain the equation for $\zeta_{i j}^{(1)}$.

$$
\Theta_{11} \tilde{\zeta}_{12}^{(1)}=\delta_{12}+\sum_{r} f_{1 r} d_{1 r 1}^{-1} \tilde{\zeta}_{r 2}^{(1)}
$$

Here the following notations are introduced

$$
\Theta_{11}=\sum_{r} \frac{1+(z-1) f_{1 r} f_{r 1}}{d_{1 r 1}} ; d_{1 r 1}=1-f_{1 r} f_{r 1}=d_{r 1 r} .
$$

Let us note that (3.26) is the equation for "correlation function" $\tilde{\zeta}_{i j}^{(1)}$ of the Ornstein-Zernike type. If we take into account the relation $\mathcal{F}_{i j}^{(2)}=$ $F_{i}^{(2)} \zeta_{i j}^{(1)}$ we obtain the equation of the present type for pair $\mathrm{CF} \mathcal{F}_{i j}^{(2)}$. In the works $[32,33]$ the equation of this type was constructed artificially. This fact leads to unselfconsistent results for static susceptibility obtained from CFs and from free energy in the case of ordered phase. For uniform field the following relations take place

$$
\begin{aligned}
& f_{1 r}=f \pi_{1 r} ; d_{1 r 1}=d=1-f^{2} ; \Theta_{11}=\Theta=\frac{1+(z-1) f^{2}}{1-f^{2}} ; \\
& F^{20}=\frac{1+a^{2}+2 a \cosh 2 \beta \tilde{\tilde{\zeta}}}{[a+\cosh 2 \beta \tilde{\tilde{\zeta}}]^{2}} ; F^{11}=\frac{1-a^{2}}{[a+\cosh 2 \beta \tilde{\tilde{\zeta}}]^{2}} ; \\
& f=\frac{1-a^{2}}{1+a^{2}+a \cosh 2 \beta \tilde{\tilde{\zeta}}} \stackrel{D=1}{\rightarrow} \frac{\cosh (\beta \zeta)-r}{\cosh (\beta \zeta)+r}
\end{aligned}
$$

Performing Fourier-transformation for (3.26) and taking into account (3.23a) we obtain the expression for the pair CF of IM in TPCA

$$
\mathcal{F}_{(\mathbf{q})}^{(2)}=F^{(2)} \frac{1+f}{1-(z-1) f+(1-f)^{-1} f z \Theta(\mathbf{q})}=\frac{\mathcal{F}_{(0)}^{(2)}}{1+\Phi z \Theta(\mathbf{q})} .
$$


Here the following notations are introduced

$$
\begin{aligned}
& \mathcal{F}_{(0)}^{(2)}=\frac{F^{(2)}(1+f)}{1-(z-1) f} ; \quad \Phi=\frac{f(1-f)^{-1}}{1-(z-1) f} \\
& \Theta(\mathbf{q})=\frac{1}{z}[\pi(0)-\pi(\mathbf{q})]=\frac{2}{D} \sum_{i=1}^{D} \sin ^{2}\left(q_{i} / 2\right) \\
& \pi(\mathbf{q})=\sum_{r} \pi(\mathbf{r}) e^{-i \mathbf{q r}}=2 \sum_{i=1}^{D} \cos q_{i} .
\end{aligned}
$$

Let us note that equation (3.26) in site space is exact for the tree-type lattice. But for Fourier-transformation (3.30) we use the translational symmetry. Thus the expression (3.29) is exact only for $1 \mathrm{D}$ case. It leads to the well known results, which we write in both wave and site spaces $\left(\mathbf{q}=\mathbf{q}_{1}\right)[46-48]$

$$
\mathcal{F}_{(q)}^{(2)}=\frac{\cosh \beta \zeta a^{2} r^{-1}}{r^{2}+\left(1-a^{2}\right) \sin ^{2} q / 2} ; \quad\left\langle S_{0} S_{n}\right\rangle=\left(\frac{a}{r}\right)^{2} f^{n}
$$

The expression for $\left\langle S_{0} S_{n}\right\rangle$ is also obtained in the work [35] in another way.

Now we are going to consider how to obtain the equation for $\zeta_{i j l}^{(2)}$. It will give us the possibility to calculate the ternary CF. Differentiating (3.26) one finds

$$
\begin{aligned}
& \Theta_{11} \tilde{\zeta}_{123}^{(2)}=-\sum_{r} \frac{f_{1 r 3}^{(1)} f_{r 1}+f_{1 r} f_{r 13}^{(1)}}{d_{1 r 1}^{2}} \tilde{\zeta}_{12}^{(1)}+ \\
& +\sum_{r} \frac{f_{1 r 3}^{(1)}+f_{r 1}^{2} f_{r 13}^{(1)}}{d_{1 r 1}^{2}} \tilde{\zeta}_{r 2}^{(1)}+\sum_{r} \frac{f_{1 r}}{d_{1 r 1}} \tilde{\zeta}_{r 23}^{(2)}
\end{aligned}
$$

Here the following notations are used

$$
f_{1 r 3}^{(1)}=\frac{\delta}{\delta \zeta_{3}} f_{1 r} ; \quad f_{r 13}^{(1)}=\frac{\delta}{\delta \zeta_{3}} f_{r 1} .
$$

The derivatives (3.33) one can easily compute:

$$
\begin{aligned}
& f_{1 r 3}^{(1)}=f_{1 r}^{(01)} d_{1 r 1}^{-1}\left[\tilde{\zeta}_{r 3}^{(1)}-f_{r 1} \tilde{\zeta}_{13}^{(1)}\right] \\
& f_{r 13}^{(1)}=f_{r 1}^{(01)} d_{1 r 1}^{-1}\left[\tilde{\zeta}_{13}^{(1)}-f_{1 r} \tilde{\zeta}_{r 3}^{(1)}\right]
\end{aligned}
$$

where

$$
\begin{aligned}
& f_{i j}^{(01)}=\frac{\partial}{\partial_{j} \tilde{\zeta}_{i}} f_{i j}=-2 f_{i j}\left[f_{i j}^{(01)}-f_{i j} F_{i j}^{(10)}\right], \\
& f_{1 r}^{(10)}=\frac{\partial}{\partial_{r} \tilde{\zeta}_{1}} f_{1 r}=0 ; \quad f_{r 1}^{(10)}=\frac{\partial}{\partial_{1} \tilde{\zeta}_{r}} f_{r 1}=0 .
\end{aligned}
$$


Substituting (3.35) into (3.32) one can find the equation for ternary "CF" $\zeta_{i j l}^{(2)}$

$$
\begin{aligned}
& \Theta_{11} \tilde{\zeta}_{123}^{(2)}=\sum_{r} \frac{f_{1 r}}{d_{1 r 1}} \tilde{\zeta}_{r 23}^{(2)} \\
& +\sum_{T}\left\{A_{1 r} \tilde{\zeta}_{12}^{(1)} \tilde{\zeta}_{13}^{(1)}+B_{1 r}\left[\tilde{\zeta}_{12}^{(1)} \tilde{\zeta}_{r 3}^{(1)}+\tilde{\zeta}_{13}^{(1)} \tilde{\zeta}_{r 2}^{(1)}\right]-C_{1 r} \tilde{\zeta}_{r 2}^{(1)} \tilde{\zeta}_{r 3}^{(1)}\right\}
\end{aligned}
$$

Here the notations are introduced

$$
\begin{gathered}
A_{1 r}=\left[f_{1 r}^{(01)} f_{r 1}^{2}-f_{r 1}^{(01)} f_{1 r}\right] d_{1 r 1}^{-3} \\
B_{1 r}=\left[f_{r 1}^{(01)} f_{1 r}^{2}-f_{1 r}^{(01)} f_{r 1}\right] d_{1 r 1}^{-3} \\
C_{1 r}=\left[f_{r 1}^{(01)} f_{1 r}^{3}-f_{1 r}^{(01)}\right] d_{1 r 1}^{-3}
\end{gathered}
$$

Taking into consideration (3.28) the equation (3.36) for uniform field can be written in the form

$$
\begin{aligned}
& \Theta \tilde{\zeta}_{123}^{(2)}=\frac{f}{d} \sum_{r} \pi_{1 r} \tilde{\zeta}_{r 23}^{(2)} \\
& +\sum_{r} \pi_{1 r}\left\{A\left[\tilde{\zeta}_{12}^{(1)} \tilde{\zeta}_{13}^{(1)}+\tilde{\zeta}_{12}^{(1)} \tilde{\zeta}_{r 3}^{(1)}+\tilde{\zeta}_{13}^{(1)} \tilde{\zeta}_{r 2}^{(1)}\right]-C_{1 r} \tilde{\zeta}_{r 2}^{(1)} \tilde{\zeta}_{r 3}^{(1)}\right\},
\end{aligned}
$$

where

$$
A=\frac{2 m f^{2}}{(1+f)^{2} d} ; \quad C=\frac{2 m f\left(1+f+f^{2}\right)}{(1+f)^{2} d}
$$

Note that (3.38) is the three-particle analogy of Ornstein-Zernike equation. Performing Fourier-transformation in (3.38) and using the relation

$$
\Theta-\frac{f}{d} \pi\left(\mathbf{q}_{1}+\mathbf{q}_{2}\right)=\frac{1}{\tilde{\zeta}^{(1)}\left(\mathbf{q}_{1}+\mathbf{q}_{2}\right)}
$$

we get the following result for $\tilde{\zeta}^{(2)}\left(q_{1}, q_{2}\right)$

$$
\begin{aligned}
\tilde{\zeta}^{(2)}\left(\mathbf{q}_{1} \mathbf{q}_{2}\right)=\left\{\left[z+\pi\left(\mathbf{q}_{1}\right)\right.\right. & \left.\left.+\pi\left(\mathbf{q}_{2}\right)\right] A-C \pi\left(\mathbf{q}_{1}+\mathbf{q}_{2}\right)\right\} \times \\
& \times \tilde{\zeta}^{(1)}\left(\mathbf{q}_{1}\right) \tilde{\zeta}^{(1)}\left(\mathbf{q}_{2}\right) \tilde{\zeta}^{(1)}\left(\mathbf{q}_{1}+\mathbf{q}_{2}\right) .
\end{aligned}
$$

Substituting (3.41) in (3.23b) for ternary $\operatorname{CFF}^{(3)}\left(\mathbf{q}_{1}, \mathrm{q}_{2}\right)$ we finally find [35]

$$
\begin{array}{r}
\mathcal{F}^{(2)}\left(\mathbf{q}_{1} \mathbf{q}_{2}\right)=\left\{F^{(3)}+F^{(2)}\left[\left(z+\pi\left(\mathbf{q}_{1}\right)+\pi\left(\mathbf{q}_{2}\right)\right) A-\right.\right. \\
\left.\left.-C \pi\left(\mathbf{q}_{1}+\mathbf{q}_{2}\right)\right] \tilde{\zeta}^{(1)}\left(\mathbf{q}_{1}+\mathbf{q}_{2}\right)\right\} \tilde{\zeta}^{(1)}\left(\mathbf{q}_{1}\right) \tilde{\zeta}^{(1)}\left(\mathbf{q}_{2}\right)
\end{array}
$$

It is easy to see (invoking (3.39)) that at $T>T_{c}$ the relation $\mathcal{F}^{(3)}\left(\mathbf{q}_{1}, \mathbf{q}_{2}\right)=0$ fulfils. For computation of $\zeta_{123}^{(2)}, \mathcal{F}_{123}^{(3)}$ it is necessary to transform (3.41), (3.42) to site representation. The expressions for them (see [35]) will contain the sums over sites. In the $1 D$ case these sums due to the form $\zeta_{12}^{(1)}=f^{|1-2|}$ 
lead to the sums of geometrical progression. After cumbersome transformation the following expression for ternary CF can be obtained [35]

$$
F^{(3)}(j, k)=\left\langle S_{0} S_{j} S_{k}\right\rangle^{c}=F^{(3)} f^{k} ; \tilde{\zeta}^{(2)}(j, k)=2 m f^{k}\left(f^{j}-1\right) ; 0 \leq j \leq k .
$$

It is important to note that $\mathcal{F}^{(3)}(j, k)$ does not depend on intermediate index $j(0 \leq j \leq k)$. Let us note that the expression for $\mathcal{F}^{(3)}(j, k)$ which is obtained by $\bar{u}$ in the frame of TPCA coincides with the result of works $[47,48]$. In these works the explicit expressions for mentioned above quantities are written only at $k=j+1, j+2$. In the work [35] using the solution for the fields ${ }_{r} \varphi^{(2)}(j, k)$ the expression (3.43) is obtained immediately in the site representation.

\subsection{Relaxational dynamics}

In the present subsection we shall consider the relaxational dynamics of the IM. Our main task is the calculation of the longitudinal dynamic susceptibility of the system under consideration

$$
\begin{aligned}
& \chi(\mathbf{q}, \omega)=\int_{-\infty}^{+\infty} d t e^{-i \omega t} \sum_{i-j} e^{i \mathbf{q} \mathbf{R}_{i j}} \chi_{i j}(t) \\
& \chi_{i j}\left(t, t^{\prime}\right)=\left.\frac{\delta m_{i t}}{\delta \zeta_{j t^{\prime}}}\right|_{\delta \zeta_{j t^{\prime}}=0} ; m_{i t}=\left\langle S_{i}\right\rangle_{\rho(t)}=m_{i}+\delta m_{i t} .
\end{aligned}
$$

Here we consider the dynamical response of the system with respect to small time-dependent mechanical perturbation of the initial Hamiltonian in the form $\sum_{j} \delta \zeta_{i}(t) S_{j}^{z}$. For the system described by the Hamiltonian (3.1) we can calculate only the static susceptibility $\chi(\mathbf{q}, 0)$. The evaluation of $d y-$ namic susceptibility can be accomplished if we shall take aditional to (3.1) items which contain other component of pseudospins or if we shall take into account the interaction of the IM with dissipative subsystem (thermostat) [48]. Further we shall investigate the system which contains becides the (3.1) with the substitution $\zeta_{i} \rightarrow \zeta_{i, t}=\zeta_{i}+\delta \zeta_{i, t}$ also the Hamiltonian of thermostat and interaction of thermostat with the Ising subsystem in the form $\sum_{j a} U_{j}^{a} S_{j}^{a}(a=x, y, z)$. Here $U_{j}^{a}$ contains only the variables of thermostat. Within the framework of nonequilibrium statistical operator (NSO) method one can obtain [48-51] the kinetic equation (KE) for unary CF $\left\langle S_{i}\right\rangle_{\rho(t)}$. Using some approximations [48] this KE can be lead to the Glauber-type form $[52,46]$

$$
\begin{aligned}
& {\left[1+\tau_{i} \Omega_{i t}\right]\left\langle S_{i}\right\rangle_{\rho(t)}=\left\langle F^{(1)}\left(\epsilon_{i t}\right)\right\rangle_{\rho(t)},} \\
& \Omega_{i t}=\frac{d}{d t}+\zeta_{i} / \tau_{i} \frac{d^{2}}{d t^{2}} ; \quad \epsilon_{i t}=\zeta_{i t}+\sum_{r} K_{1 r} S_{r} .
\end{aligned}
$$

Here $\tau_{i}=\gamma_{i}^{-1}$ is the bare time of relaxation ( $\gamma_{i}$ is the bare damping) which depends on the parameters of thermostat and on parameters of interaction of pseudospin subsystem with the thermostat. In addition to [48] we introduce also the bare resonance frequency $\omega_{i}\left(\zeta_{i}=\omega_{i}^{-2}\right)$. This is entirely in the spirit of phenomenological equation for damped oscilator (see [5]) with frequency $\omega_{i}$ and with the damping $\gamma_{i}$. The equation $(3.45 \mathrm{a})$ in the case $\zeta_{i}=0$ can be 
obtained also if we shall proceed from the Glauber master equation for the density matrix of the system $[52,46]$. If we shall develop $F^{(1)}\left(\epsilon_{i t}\right)$ as a set in $S_{r}$ one can obtain expression for $\left\langle F^{(1)}\left(\epsilon_{i t}\right)\right\rangle$ which contains distribution functions starting from unary $\left\langle S_{i}\right\rangle_{\rho(t)}$ up to the function of the power $z$ $\left\langle S_{i_{1}} S_{i_{2}} \cdots S_{i_{z}}\right\rangle_{\rho(t)}$. The site indices of mentioned above functions belong to the certain coordinate sphere $\pi_{i}$ of the site $i$. In the following for average $\left\langle F^{(1)}\left(\epsilon_{i t}\right)\right\rangle$ we shall restrict ourselves only by one-particle approximation

$$
\begin{aligned}
& \left\langle F^{(1)}\left(\epsilon_{i t}\right)\right\rangle_{\rho_{(t)}} \approx F^{(1)}\left(\tilde{\zeta}_{i t}\right), \\
& \tilde{\zeta}_{i t}=\zeta_{i t}+\Delta_{i t} ; \Delta_{i t}=\sum_{r \in \pi_{i}} r \varphi_{i t}=\sum_{r \in \pi_{i}} K_{i r}{ }^{\varphi}\left\langle S_{r}\right\rangle_{t}
\end{aligned}
$$

and two-particle one

$$
\begin{aligned}
& \left\langle F^{(1)}\left(\epsilon_{i t}\right)\right\rangle_{\rho_{(t)}} \approx\left\langle F^{(1)}\left({ }_{j} \tilde{\zeta}_{i t}+K_{i j} S_{j}\right)\right\rangle_{\rho_{(t)}} \equiv \\
& \equiv L_{i}\left(\tilde{\zeta}_{i t}, K_{i j}\right)+P\left({ }_{j} \tilde{\zeta}_{i t}, K_{i j}\right){ }^{k}\left\langle S_{j}\right\rangle_{t}, \\
& \tilde{\zeta}_{i t}=\tilde{\zeta}_{i t}-{ }_{j} \varphi_{i t}=\zeta_{i t}+\sum_{r \in \pi_{i} ; \neq j} r \varphi_{i t} .
\end{aligned}
$$

Here the following notations are introduced

$$
\begin{aligned}
& L\left({ }_{j} \tilde{\zeta}_{i t}, K_{i j}\right)=L_{i j, t}=\frac{1}{2} \sum_{\sigma= \pm 1} F^{(1)}\left({ }_{j} \tilde{\zeta}_{i t}+K_{i j} \sigma\right) \\
& P\left({ }_{j} \tilde{\zeta}_{i t}, K_{i j}\right)=P_{i j, t}=\frac{1}{2} \sum_{\sigma= \pm 1} \sigma F^{(1)}\left({ }_{j} \tilde{\zeta}_{i t}+K_{i j} \sigma\right) .
\end{aligned}
$$

The field ${ }_{r} \varphi_{i t}$ is taken to be the same in both approximations. The equations for ${ }_{r} \varphi_{i t}$ (or for $\left\langle S_{i}\right\rangle_{t}$ ) will be written below. The direct evaluation gives the following results $\left(a_{i j}=\exp \left(-2 \beta K_{i j}\right)\right)$ :

$$
\begin{aligned}
& L_{i j, t}=\frac{\sinh \left[2 \beta_{j} \tilde{\zeta}_{i t}\right]}{\cosh \left[2 \beta_{j} \tilde{\zeta}_{i t}\right]+\cosh 2 \beta K_{i j}}= \\
& =\frac{2 a_{i j} \sinh \left[2 \beta_{j} \tilde{\zeta}_{i t}\right]}{1+a_{i j}^{2}+2 a_{i j} \cosh \left[2 \beta_{j} \tilde{\zeta}_{i t}\right]}, \\
& P_{i j, t}=\frac{\sinh 2 \beta K_{12}}{\cosh \left[2 \beta_{j} \tilde{\zeta}_{i t}\right]+\cosh 2 \beta K_{i j}}= \\
& =\frac{1-a_{i j}^{2}}{1+a_{i j}^{2}+2 a_{i j} \cosh \left[2 \beta_{j} \tilde{\zeta}_{i t}\right]}, \\
& P_{i j, t}=\left.\frac{F^{(11)}\left({ }_{j} \tilde{\zeta}_{i t},{ }_{i} \tilde{\zeta}_{j t}\right)}{\left.F^{(20)}{ }_{j} \tilde{\zeta}_{i t},{ }_{i} \tilde{\zeta}_{j t}\right)}\right|_{\zeta_{i j}=\zeta} \rightarrow f .
\end{aligned}
$$

In the last relation we take into account the expressions for intracluster CF $F_{i j}^{(11)}, F_{i j}^{(20)}$ (see (3.28a)). Substituting (3.46a) and (3.47a) at $i=1,2$ 
in (3.45a) and writing two-site systems of equations in the matrix form we obtain $\left({ }^{1} m_{i t}={ }^{1}\left\langle S_{i}\right\rangle_{t} ;{ }^{k} m_{i t}={ }^{k}\left\langle S_{i}\right\rangle_{t}\right)$

$$
\begin{aligned}
& \tau_{i} \Omega_{i t}{ }^{1} m_{i t}=F^{(1)}\left(\tilde{\zeta}_{i t}\right)-{ }^{1} m_{i t} \\
& \hat{d}_{t}{ }^{k} \mathbf{m}_{t}=\mathbf{L}_{t}-\hat{\mathcal{P}}_{t}{ }^{k} \mathbf{m}_{t} ; \quad \hat{d}_{t}=\left\{\delta_{i j} \tau_{i} \Omega_{i t}\right\} \\
& { }^{k} \mathbf{m}_{t}=\left(\begin{array}{c}
{ }^{k} \mathbf{m}_{1 t} \\
{ }^{k} \mathbf{m}_{2 t}
\end{array}\right) ; \mathbf{L}_{t}=\left(\begin{array}{c}
L_{12, t} \\
L_{21, t}
\end{array}\right) ; \hat{P}_{t}=\left(\begin{array}{cc}
1 & -P_{12, t} \\
-P_{12, t} & 1
\end{array}\right)
\end{aligned}
$$

By the direct computation one can obtain the following relations

$$
\begin{gathered}
\mathbf{F}_{t}^{10}=\mathcal{P}_{t}^{-1} \mathbf{L}_{t} ; \quad D_{t}=1-P_{12, t} P_{21, t} \\
\left(\begin{array}{c}
F_{12, t}^{10} \\
F_{21, t}^{10}
\end{array}\right)=\frac{1}{D_{t}}\left(\begin{array}{l}
L_{12, t}+P_{12, t} L_{21, t} \\
L_{21, t}+P_{21, t} L_{12, t}
\end{array}\right) .
\end{gathered}
$$

Here the function $F_{12, t}^{(10)}=F^{(10)}\left({ }_{2} \tilde{\zeta}_{1 t}, \tilde{\zeta}_{2 t}\right)$ already was used by us in the subsection 3.2 . The system (3.50) can be represented in the form

$$
\begin{aligned}
& \tau_{1} \Omega_{i t}^{1} m_{i t}=F_{i, t}^{(1)}-{ }^{1} m_{i, t} \\
& D_{t}^{-1}\left[\tau_{1} \Omega_{i t}{ }^{k} m_{i t}+\mathcal{P}_{i j, t} \tau_{j} \Omega_{j t}{ }^{k} m_{j t}\right]=F_{i j, t}^{10}-{ }^{k} m_{i, t} .
\end{aligned}
$$

The dynamic version of TPCA is constructed similarly to the static case. Thus the equality ${ }^{1} m_{i t}={ }^{k} m_{i t}$ gives the equation for the fields $j \varphi_{i t}$.

For the static fields $\zeta_{i t}=C_{i}$ the left side of $(3.52)$ is equal to zero and we obtain the system of equations $(3.8)$ for the nonfluctuating part $m_{i}$. Let us represent the effective fields in the form of sum of static and fluctuating parts.

$$
\begin{aligned}
& \zeta_{i t}=\zeta_{i}+\delta \zeta_{i t} ; \quad{ }_{j} \varphi_{i}={ }_{j} \varphi_{i}+\delta_{j} \varphi_{i t} \\
& \tilde{\zeta}_{i t}=\tilde{\zeta}_{i}+\delta \zeta_{i t} ; \quad \tilde{\zeta}_{i t}={ }_{j} \tilde{\zeta}_{i}+\delta_{j} \tilde{\zeta}_{i t} \\
& \delta_{j} \tilde{\zeta}_{i t}=\delta \tilde{\zeta}_{i t}-\delta_{j} \varphi_{i t} ; \quad \sum_{j \in \pi_{i}} \delta_{j} \varphi_{i t}=\delta \tilde{\zeta}_{i t}-\delta \zeta_{i t} .
\end{aligned}
$$

Let us substitute (3.53) in (3.52) and obtain two systems of equations. The first system of equations for $m_{i}$ coincides with (3.8) and the second one for $\delta m_{i t}$ has the form

$$
\begin{array}{r}
\tau_{i} \Omega_{i t} \delta m_{i t}=F_{i}^{(2)} \delta \tilde{\zeta}_{i t}-\delta m_{i t}, \\
D_{t}^{-1}\left[\tau_{1} \Omega_{i t} \delta m_{i t}+P_{i j} \tau_{j} \Omega_{j t} \delta m_{j t}\right]= \\
=F_{i j}^{(20)}\left[\delta \tilde{\zeta}_{i t}-\delta_{j} \varphi_{i t}\right]+F_{i j}^{(11)}\left[\delta \tilde{\zeta}_{j t}-\delta_{i} \varphi_{j t}\right]-\delta m_{i, t}
\end{array}
$$

Here the fact that in TCPA $\delta m_{i t}=\delta^{1} m_{i t}=\delta^{k} m_{i t}$ is taken into account. Using (3.54a) the terms of type $\tau_{i} \omega_{i t} \delta m_{i t}$ can be excluded from (3.54b). As the result we obtain the relationship which connects $\delta_{j} \varphi_{i t}, \delta_{i} \varphi_{j t}, \delta \zeta_{i t}$, $\delta m_{i t}$. Just one more analogous equation can be obtained by permutation of indices $i \leftrightarrow j$. From this system we express the field $\delta_{j} \varphi_{i t}$ via $\delta \zeta_{i t}$ and $\delta m_{i t}$. 
Using relation (3.53b) we obtain the equation which one can write together with (3.54a) in the case of uniform field in the following form $\left(\tau_{i}=\tau_{G}\right)$

$$
\begin{aligned}
& F^{(20)} \delta \tilde{\zeta}_{i t}=\left[1+\tau_{G} \Omega_{t}\right] \delta m_{i t} \\
& {\left[1+(z-1) f^{2} F^{(20)}\right] \delta \tilde{\zeta}_{i t}=} \\
& =F^{(20)}\left(1-f^{2}\right) \delta \zeta_{i t}+f \sum_{r \in \pi_{i}} \pi_{i r} \delta m_{r t} .
\end{aligned}
$$

Substituting $F^{(20)} \delta \hat{\zeta}_{\text {it }}$ from $(3.55 \mathrm{a})$ in $(3.55 \mathrm{~b})$ we obtain the closed set of equations for $\delta m_{i t}$. Differentiating the last equation with respect to $\zeta_{j t}$ and using (3.44) we find the expression for dynamic susceptibility of the reference system

$$
\chi(\mathbf{q}, \omega)=\frac{\chi(\mathbf{q})}{1+\Omega(\omega) \tau(\mathbf{q})} ; \Omega(\omega)=i \omega\left[1+\frac{\zeta}{\tau_{G}} i \omega\right],
$$

where $\chi(\mathbf{q})=\mathcal{F}^{(2)}(\mathbf{q})($ see $(3.29),(3.30))$ and

$$
\tau(\mathbf{q})=\frac{\tau(0)}{1+\Phi z \Theta(\mathbf{q})} ; \tau(0)=\tau_{G} \frac{1+(z-1) f^{2}}{(1-f)[1-(z-1) f]} .
$$

In the $1 \mathrm{D}$ case $(z=2, J(q)=0)$ when the fields $\zeta$ are absent $(\zeta=0)$ from (3.56) (at $\zeta=0$ ) one obtains the expression which coincides with the well-known exact solution of kinetic equation (3.45) (see [46,53]). This one gives $\left(a=\exp (-2 \beta K), q=q_{1}\right)$

$$
\chi(\mathbf{q})=\frac{a}{a^{2}+\left(1-a^{2}\right) \sin ^{2} q / 2} ; \quad \tau(\mathbf{q})=\frac{\tau_{G}}{2} \frac{1+a^{2}}{a^{2}+\left(1-a^{2}\right) \sin ^{2} q / 2} .
$$

In the work [54] the cluster solution of $(3.45)(\zeta=0)$ for $\chi(\mathbf{q}, \omega)$ at arbitrary value of $z$ was presented only at $\zeta=0, \mathrm{q}=0$, and this result coincides with (3.56). Let us now take into account the long-range interaction within MFA. Now, as before, the equation for $m_{i t}=\left\langle S_{i}\right\rangle_{\rho(t)}$ has the form (3.45a) but we use the system (3.55) in which $\delta \zeta_{i t}=\delta h_{i t}+\sum_{k} J_{i k} \delta m_{k t}$. We find the susceptibility ${ }^{k} \chi(q, \omega)$ with the aid of derivative ${ }^{L} \chi_{i j}\left(t-t^{\prime}\right)=\delta m_{i t} / \delta h_{i t^{\prime}}$ at $\delta h_{i t}=0$. This one leads to the relations of (2.7) and (3.56) type

$$
{ }^{L} \chi(\mathbf{q}, \omega)=\frac{\chi(\mathbf{q}, \omega)}{1-J(\mathbf{q}) \chi(\mathbf{q}, \omega)}=\frac{L_{\chi(\mathbf{q})}}{1+\Omega(\omega)^{L} \tau(\mathbf{q})} .
$$

In the expressions for ${ }^{L} \chi(\mathbf{q})$ and ${ }^{L} \tau(\mathbf{q})$ it is convenient to separate the part that is independent on wave vector

$$
\begin{aligned}
& { }^{L} \chi(\mathbf{q})=\frac{{ }^{L} \chi(0)}{1+{ }^{L} \Phi(\mathbf{q})} ; \quad L_{\tau}(\mathbf{q})=\frac{{ }^{L} \tau(0)}{1+{ }^{L} \Phi(\mathbf{q})} ; \\
& { }^{L} \boldsymbol{\Phi}(\mathbf{q})={ }^{L} \boldsymbol{\Phi} z \Theta(\mathbf{q})+{ }^{L} \chi(0)\left[J_{0}-J(\mathbf{q})\right] ; J_{0}=J(\mathbf{q} \rightarrow \mathbf{0}),
\end{aligned}
$$

where

$$
\begin{aligned}
& { }^{L} \chi(0)=\frac{F^{20}(1+f)}{\mathcal{D}} ;{ }^{L} \tau\left(0=\frac{1+(z-1) f^{2}}{(1-f) \mathcal{D}} ;{ }^{L} \Phi=\frac{f}{(1-f) \mathcal{D}}\right. \\
& \mathcal{D}=1-(z-1) f-J_{0} F^{20}(1+f) .
\end{aligned}
$$

If $q \rightarrow 0$ the expressions (3.60) coincide with the results of the works $[55,56]$. 


\section{Application of the two-particle cluster approx- imation to the Ising model with the arbitrary value of spin}

In the present section we shall consider the Ising-type model with the arbitrary value of spin $M$ taking into account only pair interaction $(W(\{S\})=$ $0)$. The series of concrete results we shall obtain at $M=2$ for the BlumeEmery-Griffits (BEG) model:

$$
H\left(\{\zeta\}=\sum_{i}\left[\zeta_{i} S_{i}+\zeta_{i}^{\prime} S_{i}^{2}\right]+\sum_{(i j)}\left[K S_{i} S_{j}+K^{\prime} S_{i}^{2} S_{j}^{2}\right]\right.
$$

which was suggested in the work [57] for the investigations of phase transition in mixture of $H e^{3}-H e^{4}$. This model corresponds to the system with the Hamiltonian (2.1) at $M=2$ in the case $K_{i j}^{(\mu \nu)}=\delta_{\mu \nu} K^{(\mu)} \pi_{i j}, J_{i j}^{(\mu \nu)}=$ $\delta_{\mu \nu} J^{(\mu)}$. In what follows below we do not write the index 1 and instead of index 2 we use the prime symbol. Let us note, that at $\zeta^{\prime}=-\frac{8}{3} z K^{\prime}$ the Hamiltonian (4.1) describes the system with the bilinear interaction $K S_{i} S_{j}$, with the quadrupolar interaction $K^{\prime}\left(S_{i}^{2}-8 / 3\right)\left(S_{j}^{2}-8 / 3\right)$ and with the external field $\zeta[2]$. Let us note that thermodynamic properties of the BEG model were investigated by various methods [2] including TPCA. However the calculation of correlattors have not been carried out untill present time. We will computate here within TPCA the pair CFs for the IM with arbitrary value of spin [37].

As it was noted in TCPA we can use the relation (2.22)-(2.26) with $s_{i}=z, b_{f f^{\prime}}=1, W(\{R\})=0 . R=(12)$. Now the $\mathcal{F}$-function of the system under consideration takes the iorm

$$
\mathcal{F}\left(\left\{\zeta^{(\cdot)}\right\}\right)=(1-z) \sum_{1} F_{1}\left(\left\{\tilde{\zeta}_{1}^{(\cdot)}\right\}\right)+\frac{1}{2} \sum_{1, r} F_{1 r}\left(\left\{{ }_{r} \tilde{\zeta}_{1}^{(\cdot)}\right\},\left\{{ }_{1} \tilde{\zeta}_{r}^{(\cdot)}\right\}\right)
$$

The one-particle Hamiltonian and two-particle one in the case of BEG model read [37]:

$$
\begin{aligned}
& H_{1}\left(S_{1}\right)=\tilde{\zeta}_{1} S_{1}+\tilde{\zeta}^{\prime} S_{1}^{2} \\
& \tilde{\zeta}_{1}=\zeta_{1}+\sum_{r^{\prime} \in \pi_{1}}{ }_{r^{\prime}} \varphi_{1} ; \tilde{\zeta}_{1}^{\prime}=\zeta_{1}^{\prime}+\sum_{r^{\prime} \in \pi_{1}} r^{\prime} \varphi_{1}^{\prime}, \\
& H_{12}\left(S_{1} S_{2}\right)={ }_{2} \tilde{\zeta}_{1} S_{1}+{ }_{2} \tilde{\zeta}_{1} S_{1}^{2}+{ }_{1} \tilde{\zeta}_{2} S_{2}+{ }_{1} \tilde{\zeta}_{2} S_{2}^{2}+K S_{1} S_{1}+K^{\prime} S_{1}^{2} S_{2}^{2}, \\
& { }_{2} \tilde{\zeta}_{1}=\tilde{\zeta}_{1}-2 \varphi_{1} ; \quad{ }_{2} \tilde{\zeta}_{1}=\tilde{\zeta}_{1}-{ }_{2} \varphi_{1}^{\prime} .
\end{aligned}
$$

Let us write the expression for intracluster $\mathcal{F}$-function of BEG model

$$
\begin{aligned}
& F_{1}\left(\tilde{\zeta}_{1}, \tilde{\zeta}_{1}^{\prime}\right)=\ln 2\left[e^{4 \tilde{\zeta}_{1}^{\prime}} \cosh 2 \tilde{\zeta}_{1}+1 / 2\right], \\
& F_{12}\left({ }_{2} \tilde{\zeta}_{1},{ }_{2} \tilde{\zeta}_{1}^{\prime} \mid{ }_{1} \tilde{\zeta}_{2},{ }_{1} \tilde{\zeta}_{2}^{\prime}\right)=\ln 2\left\{e^{4\left({ }_{2} \tilde{\zeta}_{1}^{\prime}+{ }_{1} \tilde{\zeta}_{2}^{\prime}\right)+16 K^{\prime}}\right. \\
& {\left[e^{4 K} \cosh 2\left({ }_{2} \tilde{\zeta}_{1}+{ }_{1} \tilde{\zeta}_{2}\right)+e^{-4 K^{\prime}} \cosh 2\left({ }_{2} \tilde{\zeta}_{1}-{ }_{1} \tilde{\zeta}_{2}\right)\right]+} \\
& \left.+e^{4{ }_{2} \tilde{\zeta}_{1}^{\prime}} \cosh 2{ }_{2} \tilde{\zeta}_{1}+e^{4} \tilde{\zeta}_{2}^{\prime} \cosh 2{ }_{1} \zeta_{2}+1 / 2\right\} .
\end{aligned}
$$


Similar to (3.5) we shall use the notations for derivatives

$$
\begin{aligned}
& \tilde{\zeta}\left(\begin{array}{cc}
\mu_{1} & \mu_{2} \\
i_{1} & i_{2}
\end{array}\right)=\delta_{i_{1} i_{2}} \delta_{\mu_{1} \mu_{2}}+\sum_{r \in \pi_{i_{1}}} r \varphi\left(\begin{array}{cc}
\mu_{1} & \mu_{2} \\
i_{1} & i_{2}
\end{array}\right), \\
& \tilde{\zeta}\left(\begin{array}{cc}
\mu_{1} & \mu_{2} \\
i_{1} & i_{2}
\end{array}\right)=\frac{\delta}{\delta \zeta_{i_{2}}^{\left(\mu_{2}\right)}} \tilde{\zeta}_{i_{1}}^{\left(\mu_{1}\right)} ; r \varphi\left(\begin{array}{cc}
\mu_{1} & \mu_{2} \\
i_{1} & i_{2}
\end{array}\right)=\frac{\delta \varphi_{i_{1}}^{\left(\mu_{1}\right)}}{\delta \zeta_{i_{2}}^{\left(\mu_{2}\right)}} \\
& F_{1}^{\left(k_{\nu} \nu+k_{\mu} \mu\right)}\left(\left\{\tilde{\zeta}_{1}^{(\cdot)}\right\}\right)=\frac{\partial^{k_{\mu}}}{\partial\left[\tilde{\zeta}_{1}^{(\mu)}\right]^{k_{\mu}}} \frac{\partial^{k_{\nu}}}{\partial\left[\tilde{\zeta}_{1}^{(\nu)}\right]^{k_{\nu}}} F_{1}\left(\left\{\tilde{\zeta}^{(\cdot)}\right\}\right), \\
& F_{1 r}^{\left(k_{\mu} \mu+k_{\nu} \nu \mid l_{\delta} \delta+l_{\epsilon} \epsilon\right)}\left(\left\{{ }_{r} \tilde{\zeta}_{1}^{(\cdot)}\right\} \mid\left\{{ }_{1} \tilde{\zeta}_{\tau}^{(\cdot)}\right\}\right)= \\
& \frac{\partial^{k_{\mu}}}{\partial\left[{ }_{r} \tilde{\zeta}_{1}^{(\mu)}\right]^{k_{\mu}}} \frac{\partial^{k_{\nu}}}{\partial\left[{ }_{r} \tilde{\zeta}_{1}^{(\nu)}\right]^{k_{\nu}}} \frac{\partial^{l_{\delta}}}{\partial\left[{ }_{1} \tilde{\zeta}_{r}^{(\delta)}\right]^{l_{\delta}}} \frac{\partial^{l_{\epsilon}}}{\partial\left[{ }_{1} \tilde{\zeta}_{r}^{(\epsilon)}\right]^{l_{\epsilon}}} F_{1 r}\left(\left\{{ }_{r} \tilde{\zeta}_{1}^{(\cdot)}\right\} \mid\left\{{ }_{1} \tilde{\zeta}_{r}^{(\cdot)}\right\}\right) .
\end{aligned}
$$

Using the relations (2.5) and stationarity condition $\frac{\partial}{\partial \varphi_{i}^{(\mu)}} F\left(\left\{\zeta^{(\cdot)}\right\}\right)=0$ one can find the system of $N(z+1) M$ equations in $N Z$ unknowns $\left\langle S_{1}^{\mu}\right\rangle$ and $N z M$ unknowns $r \varphi_{i}^{\mu}\left(\left\{\zeta^{(\cdot)}\right\},\left\{K^{(\cdot)}\right\}\right)$

$$
\begin{aligned}
& \mathcal{F}^{(1)}\left({ }_{1}^{\mu_{1}} ;\left\{\zeta^{(1)}\right\}\right)=\left\langle S_{1}^{\mu_{1}}\right\rangle_{\rho}=\left\langle S_{1}^{\mu_{1}}\right\rangle_{\rho_{1}}=F_{1}^{\left(\mu_{1}\right)}\left(\left\{\tilde{\zeta}_{1}^{(\cdot)}\right\}\right), \\
& F_{1}^{\left(\mu_{1}\right)}\left(\left\{\zeta_{1}^{(\cdot)}\right\}\right)=\left\langle S_{1}^{\mu_{1}}\right\rangle_{\rho_{1}}= \\
& \left.=\left\langle S_{1}^{\mu_{1}}\right\rangle_{\rho_{1 r}}=F_{1 r}^{\left(\mu_{1} \mid 0\right)}\left({ }_{r} \zeta_{1}^{(\cdot)}\right\} \mid\left\{{ }_{1} \zeta_{r}^{(\cdot)}\right\}\right) .
\end{aligned}
$$

When the long-range interaction is absent (4.6a) gives the system of equations for ${ }_{r} \varphi_{i}^{(\mu)}$ while (4.6a) gives the expression for unary correlation function $\left\langle S_{1}^{\mu}\right\rangle_{\rho}$.

The system (4.6b) is identified to $N z$ independent relations between trial one-particle $\rho_{1}\left(S_{1}\right)$ and two-particle $\rho_{1 r}\left(S_{1}, S_{r}\right)$ density matrices (see $(3.9)$ ). These relations and some other ones in which the quantities $\rho_{1}, \rho_{1 r}$ and $U_{1 r}$ have not explicit form remain the same form as in the case $M=1$ (see (3.11)-(3.13)). In particular, in the expansion of $\mathcal{F}$-function the $K$-function for the graph of (3.12) type give zero contribution. Similar to the case $M=1$ the TCPA gives exact results for responces of the IM with arbitrary $M$ on the tree-lattice. Let us note that below we shall use the relation of (3.10) type which we can write in the form

$$
\left\langle\left(S_{1}\right)^{K_{1}} \ldots\left(S_{1}^{M}\right)^{K_{M}}\right\rangle_{\rho_{1}}^{c}=\left\langle\left(S_{1}\right)^{K_{1}} \ldots\left(S^{M}\right)^{K_{M}}\right\rangle_{\rho_{1}}^{c} .
$$

Further we shall present the explicit form for series of responses of BEG model in TCPA over short-range interaction and in MFA over long-range interaction [37]. For the free energy function per site from (2.3) and (4.2)(4.4) we find

$$
\begin{aligned}
& \beta^{L} f=\frac{\beta}{2}\left[J_{0} m^{2}+J_{0}^{\prime} q^{2}\right]+\beta f\left(\zeta, \zeta^{\prime}, K, K^{\prime}\right), \\
& -\beta f=(1-z) \ln Z_{1}\left(\tilde{\zeta}, \bar{\zeta}^{\prime}\right)+\frac{z}{2} \ln Z_{12}\left(\tilde{\tilde{\zeta}}, \tilde{\zeta}^{\prime}\right) .
\end{aligned}
$$


Here the notations are used

$$
\begin{aligned}
& Z_{1}\left(\tilde{\zeta}, \tilde{\zeta}^{\prime}\right)=2 e^{4 \beta \tilde{\zeta}^{\prime}} \cosh 2 \beta \tilde{\zeta}+1, \\
& Z_{12}=2 e^{\beta\left(8 \tilde{\zeta}+16 K^{\prime}+4 K\right)}\left[\cosh 4 \beta \tilde{\tilde{\zeta}}+e^{-8 \beta K}\right]+ \\
& +4 e^{4 \beta \zeta^{\prime}} \cosh 2 \beta \tilde{\tilde{\zeta}}+1, \\
& \tilde{\zeta}=\zeta+z \varphi, \tilde{\tilde{\zeta}}=\zeta+(z-1) \varphi ; \quad \zeta=h+J_{0} m, \\
& \tilde{\zeta}^{\prime}=\zeta^{\prime}+z \varphi^{\prime}, \tilde{\tilde{\zeta}}=\zeta^{\prime}+(z-1) \varphi^{\prime} ; \quad \zeta^{\prime}=h^{\prime}+J_{0}^{\prime} q, \\
& m=\left\langle S_{i}\right\rangle, q=\left\langle S_{i}^{2}\right\rangle, J_{0}=J(\mathbf{q} \rightarrow 0), J_{0}^{\prime}=J^{\prime}(\mathbf{q} \rightarrow 0) .
\end{aligned}
$$

The system of four equations (4.6) for $m, q, \varphi, \varphi^{\prime}$ on the basis (4.4)-(4.5)can be obtained in the form

$$
\begin{aligned}
& m=\frac{4 e^{4 \beta \tilde{\zeta}} \sinh 2 \beta \tilde{\zeta}}{Z_{1}\left(\tilde{\zeta}, \tilde{\zeta}^{\prime}\right)}, \\
& q=\frac{8 e^{4 \beta \tilde{\zeta}^{\prime}} \cosh 2 \beta \tilde{\zeta}}{Z_{1}\left(\tilde{\zeta}, \tilde{\zeta}^{\prime}\right)}, \\
& \frac{e^{4 \beta \tilde{\zeta}^{\prime}} \sinh 2 \beta \tilde{\zeta}}{Z_{1}(\tilde{\zeta}, \tilde{\zeta})}= \\
& =\frac{e^{\beta\left(8 \tilde{\zeta}^{\prime}+16 K^{\prime}+4 K\right)} \sinh 4 \beta \tilde{\tilde{\zeta}}+e^{4 \beta \tilde{\tilde{\zeta}}} \sinh 2 \beta \tilde{\tilde{\zeta}}}{Z_{12}\left(\tilde{\tilde{\zeta}}, \tilde{\zeta}^{\prime}\right)} \\
& \frac{e^{4 \beta \tilde{\zeta}^{\prime}} \cosh 2 \beta \tilde{\zeta}}{Z_{1}\left(\tilde{\zeta}, \tilde{\zeta}^{\prime}\right)}= \\
& =\frac{e^{\beta\left(8\left(8 \tilde{\zeta}^{\prime}+16 K^{\prime}+4 K\right)\right.}\left(\cosh 4 \beta \tilde{\tilde{\zeta}}+e^{-8 \beta K}\right)+e^{4 \beta \tilde{\zeta}^{\prime}} \cosh 2 \beta \tilde{\tilde{\zeta}}}{Z_{12}\left(\tilde{\tilde{\zeta}}, \tilde{\tilde{\zeta}}^{\prime}\right)} .
\end{aligned}
$$

Let us note that numerical investigation of system (4.10) at different values of parameters of the Hamiltonian (4.1) in the case $J_{0}=J_{0}^{\prime}=0$ is carried out in the work [37]. Here it is also investigated the thermodynamic potential and static susceptibility of BEG model.

The method of finding CF of IM with arbitrary value $M$ is similar to one described in subsection 3.2. But now the matrix relation over indices $\mu, \nu$ takes place. One can obtain from the expression (4.6a) the matrix relations of (3.23) type connecting CF with derivatives from $\tilde{\zeta}_{i}^{(\mu)}$. In the present work we restrict ourselves to finding only pair CF. Thus differentiating (4.6b) with respect to $\zeta_{2}^{\left(\mu_{2}\right)}$ we obtain the relation of (3.25) type

$$
\begin{aligned}
& r \hat{\varphi}_{12}+\hat{f}_{1 r 1} \hat{\varphi}_{r 2}=\hat{f}_{1 r} \hat{\tilde{\zeta}}_{r 2}, \\
& \hat{f}_{r 1 r} \hat{\varphi}_{12}+{ }_{1} \hat{\varphi}_{r 2}=\hat{f}_{r 1} \hat{\tilde{\zeta}}_{12} .
\end{aligned}
$$

Here the following notations and relations are used

$$
\hat{f}_{i j}=\left[\hat{F}_{i}^{(2)}\right]^{-1} \hat{F}_{i j}^{(11)} ; \quad \hat{F}_{i}^{(2)} \equiv \hat{F}_{i j}^{(20)},
$$




$$
\begin{array}{ll}
\hat{F}_{i}^{(2)}=\left\{F^{(\mu+\nu)}\left(\left\{\tilde{\zeta}_{1}^{(\cdot)}\right\}\right)\right\} ; & \hat{F}_{i j}^{(11)}=\left\{F^{(\mu \mid \nu)}\left(\left\{_{j} \tilde{\zeta}_{i}^{(\cdot)}\right\} \mid\left\{i \zeta_{j}^{(\cdot)}\right\}\right)\right\}, \\
\hat{\zeta_{i j}}=\left\{\zeta\left(\begin{array}{cc}
\mu & \nu \\
i & j
\end{array}\right)\right\} ; & r \hat{\varphi}_{i j}=\left\{r \varphi\left(\begin{array}{cc}
\mu & \nu \\
i & j
\end{array}\right)\right\} .
\end{array}
$$

So expressing the matrices ${ }_{r} \hat{\varphi}_{i j}$ with the aid of $\hat{\bar{\zeta}}_{i j}$ and forming the closed equation for $\tilde{\vec{\zeta}}_{i j}$ one can find at the case of uniform field the expression for $\hat{\tilde{\zeta}}(\mathbf{q})$. On the basis of matrix relation of (3.23a) type we find the expression for pair $\mathrm{CF}^{(2)}(\mathbf{q})$. It can be written in two forms [37]

$$
\begin{aligned}
& \hat{\mathcal{F}}^{(2)}(\mathbf{q})=\hat{F}^{(2)}[1-(z-1) \hat{f}+ \\
& \left.(1-\hat{f})^{-1} \hat{f} z \Theta(\mathbf{q})\right]^{-1}(1+\hat{f}) \\
& {\left[\hat{\mathcal{F}}^{(2)}\right]^{-1}(\mathbf{q})=(1-z)\left[\hat{F}^{(2)}\right]^{-1}+z\left[\hat{F}^{(2)}+\hat{F}^{(11)}\right]^{-1}+} \\
& +\left[\hat{F}^{(2)}\left(\hat{F}^{(11)}\right)^{-1} \hat{F}^{(2)}-\hat{F}^{(11)}\right]^{-1} z \Theta(\mathbf{q}) .
\end{aligned}
$$

In the case of BEG model we shall present the explicit form of matrix elements (intracluster pair CF), which are included in $(4.13)\left(F^{\left(1+1^{\prime}\right)}\right.$, $\left.F^{\left(1+0^{\prime} \mid 0+1^{\prime}\right)}\right)$-nondiagonal elements and $l\left(l^{\prime}\right)$ denotes the number of derivatives with respect to unprimed (primed) field [37]:

$$
\begin{aligned}
& F^{\left(2+0^{\prime}\right)}=8 e^{4 \beta \tilde{\zeta}^{\prime}}\left[2 e^{4 \beta \bar{\zeta}^{\prime}}+\cosh 2 \beta \tilde{\zeta}\right] Z_{1}^{-2}, \\
& F^{\left(0+2^{\prime}\right)}=\frac{32 e^{4 \beta \tilde{\zeta}^{\prime}} \cosh 2 \beta \tilde{\zeta}}{Z_{1}^{2}} \\
& F^{\left(1+1^{\prime}\right)}=F^{\left(1^{\prime}+1\right)}=\frac{32 e^{4 \beta \bar{\zeta}^{\prime}} \sinh 2 \beta \tilde{\zeta}}{Z_{1}^{2}} \\
& F^{\left(1+0^{\prime} \mid 1+0^{\prime}\right)}=8 e^{8 \beta \tilde{\bar{\zeta}}}\left\{e ^ { 1 6 \beta K ^ { \prime } } \left[4 e^{\beta\left(8 \tilde{\zeta}+16 K^{\prime}\right)} \sinh 8 \beta K+\right.\right. \\
& +8 e^{4 \beta \tilde{\bar{\zeta}}^{\prime}} \sinh 4 \beta K \cosh 8 \beta \tilde{\tilde{\zeta}}+ \\
& \left.\left.+e^{4 \beta K} \cosh 4 \beta \tilde{\tilde{\zeta}}-e^{-4 \beta K}\right]-2 \sinh ^{2} 2 \beta \tilde{\tilde{\zeta}}\right\} Z_{12}^{-2} \\
& F^{\left(0+1^{\prime} \mid 0+1^{\prime}\right)}=\frac{32}{Z_{12}^{2}} e^{8 \beta \tilde{\tilde{\zeta}}^{\prime}}\left\{e^{\beta(16 K+4 K)}\left[\cosh 4 \beta \tilde{\tilde{\zeta}}+e^{-8 \beta K}\right]-2 \cosh ^{2} 2 \beta \tilde{\tilde{\zeta}}\right\}, \\
& F^{\left(1+0^{\prime} \mid 0+1^{\prime}\right)}=F^{\left(0+1^{\prime} \mid 1+0^{\prime}\right)}=\frac{16}{Z_{12}^{2}} e^{8 \beta \tilde{\zeta}^{\prime}}\left\{4 e^{\beta\left(4 \tilde{\zeta}+16 K^{\prime}\right)} \sinh 2 \beta \tilde{\tilde{\zeta}} \times\right. \\
& \left.\times \sinh 4 \beta K+e^{\beta\left(16 K^{\prime}+4 K\right)} \sinh 4 \beta \overline{\tilde{\zeta}}-2 \sinh 2 \beta \tilde{\tilde{\zeta}} \cosh 2 \beta \tilde{\tilde{\zeta}}\right\}
\end{aligned}
$$

Let us note that the form (4.13) is the typical one for TPCA. Formula (4.13) gives expression for averaging over configuration of pair spin-spin CF [58] in the case of Ising mixture with the quenched disorder of replacement. Now the indices $\mu, \nu$ number sorts of atoms; and intracluster CF 
contain averaging over sort configuration. In the case of the one-sort quantum system (the Hamiltonian of such system contains different components of spin operators $S^{a}$ ) the form (4.13) gives expressions for the pair cumulant temperature Green functions in the impulse-frequency representation $[42,43]$. Now the indices $\mu, \nu$ number the $\mathrm{x}, \mathrm{y}, \mathrm{z}$ component of spin operators; $\hat{F}^{(2)} \rightarrow \hat{F}^{(2)}\left(\omega_{n}\right), \hat{F}^{(11)} \rightarrow \hat{F}^{(11)}\left(\omega_{n}\right)$ are expressed with the aid of intracluster Green functions.

\section{Thermodynamics and pair correlation functi- ons of $\mathrm{KD}_{2} \mathrm{PO}_{4}$ ferroelectrics in four-particle cluster approximation}

\subsection{Statement of cluster expansion method. Intracluster correlations function}

We shall consider a system of deuterons moving on O-D..O bonds in compound of $\mathrm{KD}_{2} \mathrm{PO}_{4}$ type. This compound is related to orthorombic symmetry with the four molecules per West's elementary cell which is characterized by basic vector: $\left|\mathbf{e}_{1}\right|=\left|\mathbf{e}_{1}\right|=7.469 \mathrm{~A},\left|\mathbf{e}_{3}\right|=6.975 \mathrm{~A}$. The unit cell of the Bravais lattice is composed of two neighbouring $\mathrm{PO}_{4}$ tetrahedra together with four hydrogen $(\alpha=1,2,3,4)$ bond relating to one of them (A - type tetrahedron). Hydrogen bonds going to another (B - type) tetrahedron belong to four nearest structural elements surrounding it. Their coordinates are $n_{1}, n_{1}+r_{2}, n_{1}+r_{3}, n_{1}+r_{4}$. The projection of two neighbouring tetrahedra on the plane $\left(e_{1} \times e_{2}\right)$ as well as the effect of cluster fields on deuterons are presented in the figure 2 .

The Hamiltonian of deuteron subsystem taking into account short-range interaction and taking into c.nsideration long-range interaction $J_{\alpha \alpha}\left(n-n^{9}\right)$ in MFA can be written as follows $[32,33]$

$$
\begin{aligned}
& H(\{\zeta\})=\sum_{n, \alpha} \zeta_{n \alpha} S_{n \alpha}-\sum_{R} V_{R}(\{s\}), \\
& V_{R}(\{s\})=V\left(n_{1}, n_{2}, n_{3}, n_{4}\right)=\left\{\frac{1}{8} \sum_{\alpha, \alpha^{\prime}} V_{\alpha \alpha^{\prime}} S_{n_{\alpha} \alpha} S_{n_{\alpha^{\prime}} \alpha^{\prime}}+\right. \\
& \left.+\frac{1}{16} \Phi S_{n_{1} 1} S_{n_{2} 1} S_{n_{3} 3} S_{n_{4} 4}\right\}\left\{\delta_{n_{1} n_{2}} \delta_{n_{1} n_{3}} \delta_{n_{1} n_{4}}+\right. \\
& \left.+\delta_{n_{1}+r_{2}, n_{2}} \delta_{n_{1}+r_{3}, n_{3}} \delta_{n_{1}+r_{4}, n_{4}}\right\} \\
& \zeta_{n \alpha}=h_{n \alpha}+\lambda_{n \alpha} ; \lambda_{n \alpha}=\sum_{n^{\prime} \alpha^{\prime}} J_{\alpha \alpha^{\prime}}\left(n-n^{\prime}\right)\left\langle S_{n^{\prime} \alpha^{\prime}}\right\rangle .
\end{aligned}
$$

Here $S_{n \alpha}= \pm 1$ describes the position of deuteron in double well potential which situates on $\alpha$ th hydrogen bond in $n$th cell of Bravais lattice, $n_{\alpha}=n+r_{\alpha}$ where $r_{\alpha}$ is the radius-vector of the hydrogen bond $\alpha$ in the present cell $\vec{n}$. In (5.1b) the first term (the first product of Kroneker symbols) describes the short-range configurational interactions of deuterons near "A" tetrahedra, the second term describes the same interactions near "B" tetrahedra. Let us note that parameter $J_{\alpha \alpha}\left(n-n^{\prime}\right)$ includes also an indirect deuteron interaction via lattice vibration. Further we shall use the following well-known notations

$$
V_{12}=V_{23}=V_{34}=V_{41}=-\frac{1}{2} W_{1}=V_{s},
$$




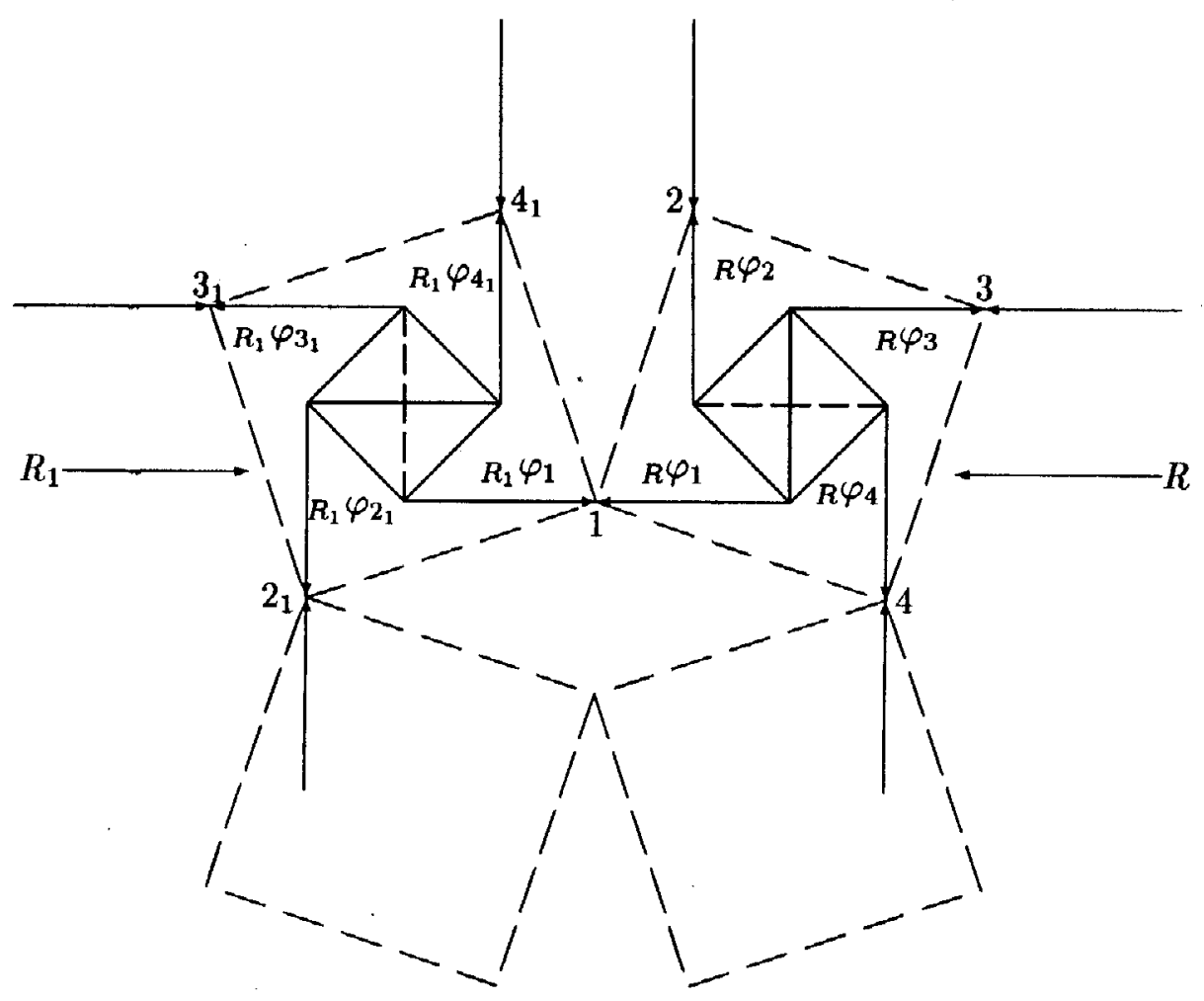

Figure 2; Two neighbouring tetrahedra ( $\mathrm{A}$ and $\mathrm{B}$ type) and lattice constructed from four-particle clusters in projection on the plane $\left(\mathbf{e}_{1} \times \mathbf{e}_{2}\right)$. The tops of clusters coincide with the centres of hydrogen bonds. The effect of cluster fields on sites of cluster lattice is denoted by pointers. The sites of cluster $R_{0}=R\left(R_{1}\right)$ are numbered by indices $i_{0}=i\left(i_{1}\right)$.

$$
\begin{aligned}
& V_{13}=V_{24}=-\epsilon+\frac{1}{2} W_{1}=U_{s}, \\
& \Phi=4 \epsilon-8 W+2 W_{1} ; \quad \epsilon=\epsilon_{a}-\epsilon_{s}, \\
& W=\epsilon_{1}-\epsilon_{s}, \quad W_{1}=\epsilon_{0}-\epsilon_{s} .
\end{aligned}
$$

Here $\epsilon_{s}, \epsilon_{1}, \epsilon_{0}, \epsilon_{a}$ are four different configurational energies for sixteen configurations of deuterons near $\left[\mathrm{PO}_{4}\right]^{3-}$ tetrahedra (see $\left.[4,5,32]\right)$. It is convenient to use the general index $i=\left(n_{i}, \alpha_{i}\right)$. Let us decompose pseudospin lattice into clusters. The cluster we shall take in the form of tetrahedron. Its tops coinelde with the centrea of hydrogen bonds. The projections of the same clusters on the plane $a \times b$ is noted by shade line (see figure 2 ). Let us note that the lattice which is formed from the same clusters is similar to deformated square lattice with chess decomposition of this lattice into square clusters. Evidently that the structure of the Hamiltonian (5.1) agrees with that of (2.1). So we shall use the relations of section 2 (starting from (2.8)) at $R=(1,2,3,4)$ when we shall perform the cluster expansion. In the present work we limit ourselves to four-particle cluster approximation (FPCA). Thus one may use relations $(2.22)-(2.26)$. For reference $\mathcal{F}$-function 
we write

$$
\mathcal{F}(\{\zeta\})=-\sum_{i} F_{i}\left(\tilde{\zeta}_{i}\right)+\sum_{R} F_{R}(\{R \tilde{\zeta}\}),
$$

where

$$
\begin{aligned}
& F_{i}\left(\tilde{\zeta}_{i}\right)=\ln Z_{i}\left(\tilde{\zeta}_{i}\right)=\ln S p e^{\bar{\zeta}_{i} S_{i}}=\ln 2 \cosh \tilde{\zeta}_{i}, \\
& F_{R}(\{R \tilde{\zeta}\})=\ln S p e^{H_{R}(\{s\})}=F_{1234}^{(0000)}= \\
& =\ln Z_{1234}^{(000)}=F_{R}\left(R_{1} \tilde{\zeta}_{1}, R_{2} \tilde{\zeta}_{2}, R_{3} \tilde{\zeta}_{3}, R_{4} \tilde{\zeta}_{4}\right)= \\
& =F_{R}\left(\tilde{\tilde{\zeta}}_{1}, \tilde{\tilde{\zeta}}_{2}, \tilde{\tilde{\zeta}}_{3}, \tilde{\tilde{\zeta}}_{4}\right), \\
& H(\{s\})=-V_{R}(\{s\})+\sum_{f \in R} R_{f} \zeta_{f} S_{f}, \\
& \tilde{\zeta}_{f}=\zeta_{f}+\sum_{R} \varphi_{f} ; \quad R_{f} \zeta_{f}=\tilde{\zeta}_{f}-R_{f} \varphi_{f}=\tilde{\tilde{\zeta}}_{f} .
\end{aligned}
$$

The explicit form of cluster partition function is the following

$$
\begin{aligned}
& \frac{1}{2} Z_{R}=\frac{1}{2} Z_{1234}^{(0)}=2 a \cosh \left(\tilde{\tilde{\zeta}}_{1}-\tilde{\tilde{\zeta}}_{3}\right) \cosh \left(\tilde{\tilde{\zeta}}_{2}-\tilde{\tilde{\zeta}}_{4}\right)+ \\
& +d \cosh \left(\tilde{\tilde{\zeta}}_{1}-\tilde{\tilde{\zeta}}_{2}+\tilde{\tilde{\zeta}}_{3}-\tilde{\tilde{\zeta}}_{4}\right)+\cosh \left(\tilde{\tilde{\zeta}}_{1}+\tilde{\tilde{\zeta}}_{2}+\tilde{\tilde{\zeta}}_{3}+\tilde{\tilde{\zeta}}_{4}\right)+ \\
& +2 b\left[\cosh \left(\tilde{\tilde{\zeta}}_{1}+\tilde{\tilde{\zeta}}_{3}\right) \cosh \left(\tilde{\tilde{\zeta}}_{2}-\tilde{\tilde{\zeta}}_{4}\right)+\right. \\
& \left.+\cosh \left(\tilde{\tilde{\zeta}}_{1}-\tilde{\tilde{\zeta}}_{3}\right) \cosh \left(\tilde{\tilde{\zeta}}_{2}+\tilde{\tilde{\zeta}}_{4}\right)\right], \\
& a=e^{-\beta_{\epsilon}} ; \quad b=0^{-\beta W} ; \quad d=e^{-\beta W_{1}} .
\end{aligned}
$$

Let us note that we neglect general factor $\exp \left\{\left(W_{1}+4 W+2 \epsilon\right) / 8\right\}$ in (5.5). It gives independent of temperature term in to free energy $-\beta^{-1} \mathcal{F}(\{\zeta\})$. Let us introduce the following notations for derivatives

$$
\begin{aligned}
& F_{i}^{(l)}\left(\tilde{\zeta}_{i}\right)=\frac{\partial^{l}}{\left(\partial \tilde{\zeta}_{i}\right)^{l}} F\left(\tilde{\zeta}_{i}\right) ; \quad F_{f, R}^{(l)}=\frac{\partial^{l}}{\left(\partial \tilde{\tilde{\zeta}}_{f}\right)^{l}} F_{1234}^{(0000)}, \\
& F_{f f^{\prime}, R}^{\left(l^{\prime}\right)}=\frac{\partial}{\partial\left(\tilde{\tilde{\zeta}}_{f}\right)^{l}} \frac{\partial}{\partial\left(\tilde{\tilde{\zeta}}_{f^{\prime}}\right)^{l \prime}} F_{1234}^{(0000)} ; f, f^{\prime}=(1,2,3,4)=(R) .
\end{aligned}
$$

The system of equations for averages $m_{i}=\left\langle S_{i}\right\rangle$ and fields $R \varphi_{i}$ is obtained as in the previous section on the basis (2.5) and stationarity condition of $\mathcal{F}(\{\zeta\})$ with respect to $R \varphi_{i}\left({ }^{L} m_{i}=m_{i}\right)$

$$
\begin{aligned}
& m_{i}={ }^{k} \mathcal{F}_{1}^{(1)}=F^{(1)}\left(\tilde{\zeta}_{1}\right)=\tanh \tilde{\zeta}_{i}, \\
& F_{1}^{(1)}\left(\tilde{\zeta}_{1}\right)=F_{1, R_{r}}^{(1)} ; \quad r=0,1 .
\end{aligned}
$$

Here index $r$ numbers two clusters $r=0,1$ which contain bond 1 (see figure 2). Therefore there are two fields per one site. Then the system (5.7b) gives $8 N$ equations in $8 N$ unknowns ${ }_{R} \varphi_{i}$ where $N$ is the number of A (or B) tetrahedron in the crystal. We have one equation (5.7b) for the 
field $\varphi$ in the case of uniform field. The system $(5.7 \mathrm{~b})$ is equivalent to the following system of equations

$$
\rho_{1}\left(S_{1}\right)=S p_{S_{2 r} S_{3 r} S_{4 r}} \rho_{R}\left(S_{1}, S_{2 r}, S_{3 r}, S_{4 r}\right)
$$

In particular from (5.8) the relation between intracluster CF follows:

$$
\left\langle S_{1}^{(}\right\rangle_{\rho_{1}}^{c}=F_{1}^{(l)}\left(\tilde{\zeta}_{1}\right)=F_{1, R}^{(l)}=\left\langle S_{1}^{l}\right\rangle_{\rho_{R}}^{c}
$$

One can obtain the relation for moment function from (5.8)

$$
M(R)=S p_{1,2,3,4} \rho_{1} \rho_{2} \rho_{3} \rho_{4} e^{U_{R}}=S p_{2,3,4} \rho_{2} \rho_{3} \rho_{4} e^{U_{R}}
$$

One can carry out the investigation of diagrams for $F$-function and $\left\langle S_{1}\right\rangle=$ $\frac{\delta}{\delta \zeta_{1}} F$ in the higher order of cluster expansion similar as it was done in the case of two-particle cluster. This investigation leads to conclusion that FPCA gives the exact results for all characteristics of the system with the Hamiltonian (5.1) in the case of tree-like lattice. This lattice is constructed on the basis of tetrahedra. It can be formed from two-particle tree with $z=4$ if all sites will change by tetrahedra. Now let us write some results in the homogeneous field. The free energy per tetrahedron in FPCA taking into account long-range interaction in MFA reads $\left(m_{\alpha}=m\right)$ :

$$
\begin{aligned}
& f=-\frac{k T}{N} \mathcal{F}(h)=\frac{\nu_{1}\left(\hat{q}_{0}\right)}{2} m^{2}+4 k T \ln 2 \cosh \beta \tilde{\zeta}-2 k T \ln Z_{R}(\tilde{\tilde{\zeta}}) \\
& \frac{1}{2} Z_{R}(\tilde{\tilde{\zeta}})=2 a+d+4 b \cosh 2 \beta \tilde{\tilde{\zeta}}+\cosh 4 \beta \tilde{\tilde{\zeta}}
\end{aligned}
$$

The eigenvalue $\nu_{1}\left(\hat{q}_{0}\right)$ of the matrix $J_{\alpha \alpha}\left(\hat{q}_{0}\right)$ reads:

$$
\nu_{1}\left(\hat{q}_{0}\right)=J_{11}\left(\hat{q}_{0}\right)+J_{13}\left(\hat{q}_{0}\right)+2 J_{12}\left(\hat{q}_{0}\right),
$$

where $\hat{q}_{0}$ is the vector belong to the wave vector's star $\left\{q_{0}\right\}(\epsilon \rightarrow 0)$

$$
\left\{\mathbf{q}_{0}\right\}= \pm \hat{q}_{0 x}, \pm \hat{q}_{0 y} ; \hat{q}_{0 x}=(\epsilon, 0,0) ; \hat{q}_{0 y}=(0, \epsilon, 0) \text {. }
$$

Fields $\tilde{\zeta}, \tilde{\zeta}$ contain the eigenvalue $\nu_{1}\left(\hat{q}_{0}\right)$ via molecular field $\nu_{1}\left(\hat{q}_{0}\right) m$ :

$$
\tilde{\zeta}=\zeta-2 \varphi ; \tilde{\tilde{\zeta}}=\zeta-\varphi ; \zeta=h+\nu_{1}\left(\hat{q}_{0}\right) m \text {. }
$$

The system of equations for $m, \varphi$ which follows from(5.7) reads

$$
m=\tanh \beta \tilde{\zeta}=4 \sinh 2 \beta \tilde{\tilde{\zeta}}[b+\cosh 2 \beta \tilde{\tilde{\zeta}}] Z_{R}^{-1}(\tilde{\tilde{\zeta}})
$$

Evidently from the first equation of (5.15) we can obtain the expression for $\varphi=\varphi(m)$ and we can reduce (5.15) to one equation for parameter $m$.

Further we shall use also the following intracluster $\mathrm{CF}$ (we present them only at $\zeta_{i}=\zeta$ )

$$
F^{(1)}=m=\tanh \beta \tilde{\zeta} ; \quad F^{(2)}=1-m^{2},
$$




$$
\begin{aligned}
& F_{1}(\tilde{\tilde{\zeta}})=F_{12, R}^{(11)}=2 \frac{-d+\cosh 4 \beta \tilde{\tilde{\zeta}}}{Z_{R}(\tilde{\tilde{\zeta}})}-m^{2} \stackrel{T \geq T_{c}}{\rightarrow} \frac{1-d}{1+2 a+4 b+d}, \\
& F_{2}(\tilde{\tilde{\zeta}})=F_{13, R}^{(11)}=2 \frac{+d-2 a+\cosh 4 \beta \tilde{\tilde{\zeta}}}{Z_{R}(\tilde{\tilde{\zeta}})}-m^{2} \stackrel{T \geq T_{c}}{\rightarrow} \frac{1-2 a+d}{1+2 a+4 b+d}, \\
& F_{1}=F_{12, R}^{(11)}=F_{13, R}^{(11)}=F_{24, R}^{(11)}=F_{34, R}^{(11)} \\
& F_{2}=F_{13, R}^{(11)}=F_{24, R}^{(11)} .
\end{aligned}
$$

\subsection{Pair correlation functions of the reference system}

The procedure for obtaining CFs of the reference system is similar to mentioned above in the case of two-particle cluster. From (2.5) and (5.7) one has

$$
\begin{aligned}
& \left\langle S_{i} S_{j}\right\rangle=F_{i j}^{(2)}=F_{i}^{(2)} \tilde{\zeta}_{i j}^{(1)}, \\
& \tilde{\zeta}_{i j}^{(1)}=\frac{\delta}{\delta \zeta_{j}} \tilde{\zeta}_{i}=\delta_{i j}-\sum_{R^{\prime}} R^{\prime} \varphi_{i j}^{(1)} ; R^{\prime} \varphi_{i j}^{(1)}=\frac{\delta}{\delta \zeta_{j}} R^{\prime} \varphi_{i}, \\
& \tilde{\zeta}_{i j}^{(1)}=\frac{\delta}{\delta \zeta_{j}} \tilde{\zeta}_{i}=\delta_{i j}-R_{i} \varphi_{i j}^{(1)}=\tilde{\zeta}_{i j}^{(1)}+{ }_{R} \varphi_{i j} .
\end{aligned}
$$

For obtaining equation for $\tilde{\zeta}_{i j}^{(1)}$ we differentiate $(5.7 \mathrm{~b})$ with respect to $\zeta_{f}$. This leads to relation (the $\mathrm{nc}^{+}$ations $R, R_{r}, 1,2_{r}, 3_{r}, 4_{r}$ correspond to figure 2)

$$
\begin{aligned}
& F_{1}^{(2)}{ }_{R} \varphi_{1 j}^{(1)}+F_{12, R}^{(11)} \varphi_{2 j}^{(1)}+F_{13, R}^{(11)} \varphi_{3 j}^{(1)}+F_{14, R}^{(11)} \varphi_{4 j}^{(1)}= \\
& =-F_{12, R}^{(11)} \tilde{\zeta}_{2 j}^{(1)}-F_{13, R}^{(11)} \tilde{\zeta}_{3 j}^{(1)}-F_{14, R}^{(11)} \tilde{\zeta}_{4 j}^{(1)} .
\end{aligned}
$$

After cyclic permutation of the indices $1,2,3,4$, in (5.18) we shall find another three relations of (5.18) type. One can write for cluster $R_{1}$ four equation of the same type. Let us present these equations for cluster $R_{T}$ $(r=0,1)$ in the matrix form

$$
\begin{aligned}
& {\left[\hat{F}_{R_{r}}^{(2)}+\hat{F}_{R_{r}}^{(11)}\right]_{R_{r}} \varphi_{(r), j}^{(1)}=-\hat{F}_{R_{r}}^{(11)} \tilde{\zeta}_{(r), j}^{(1)},} \\
& \hat{F}^{(2)}=\left\{F_{f}^{(2)} \delta_{f f^{\prime}}\right\} ; \quad \hat{F}_{R}^{(11)}=\left\{F_{f f^{\prime}, R}^{(11)}\left(1-\delta_{f f^{\prime}}\right)\right\}, \\
& \boldsymbol{R}_{r} \hat{\varphi}_{(r), j}^{(1)}=\left\{R_{r} \varphi_{i j}^{(1)}\right\} ; \quad \hat{\tilde{\zeta}}_{(r), j}^{(1)}=\left\{\tilde{\zeta}_{i j}^{(1)}\right\} .
\end{aligned}
$$

Here $F^{(2)}, F^{(11)}$ are the square matrices. They are formed by indices $f, f^{\prime}=$ $1_{r}, 2_{r}, 3_{r}, 4_{r}$. The columnar matrices $R_{r} \varphi^{(1)}, \hat{\tilde{\zeta}}_{j}^{(1)}$ are formed by index $j=$ $1,2,3,4$.

In the following we consider only uniform case. Then on the basis of two matrix equations (5.19) (writen at $r=0$ and $r=1$ ) and after some 
transformations we obtain the matrix equation

$$
\left(\begin{array}{c}
-\tilde{\zeta}_{1 j}^{(1)}+\delta_{1 j} \\
\varphi_{2 j}^{(1)}+\varphi_{2_{1 j}}^{(1)} \\
\varphi_{3 j}^{(1)}+\varphi_{3_{1 j}}^{(1)} \\
\varphi_{4 j}^{(1)}+\varphi_{4_{1 j}}^{(1)}
\end{array}\right)=-A\left(\begin{array}{c}
2 \tilde{\zeta}_{1 j}^{(1)} \\
\tilde{\zeta}_{2 j}^{(1)}+\tilde{\zeta}_{2, j}^{(1)} \\
\tilde{\zeta}_{3 j}^{(1)}+\tilde{\zeta}_{3_{j j}}^{(1)} \\
\tilde{\zeta}_{4 j}^{(1)}+\bar{\zeta}_{4_{1 j} j}^{(1)}
\end{array}\right),
$$

where

$$
\begin{aligned}
& \hat{A}=\left[\hat{F}^{(2)}+\hat{F}^{(11}\right] \hat{F}^{(11)}, \\
& \hat{F}^{(2)}=\left\{F^{(2)} \delta_{f J^{\prime}}\right\} ; \hat{F}^{(11)}=\left(\begin{array}{cccc}
0 & F_{1} & F_{2} & F_{1} \\
F_{1} & 0 & F_{1} & F_{2} \\
F_{2} & F_{1} & 0 & F_{1} \\
F_{1} & F_{2} & F_{1} & 0
\end{array}\right) .
\end{aligned}
$$

It is necessary to obtain the closed equation for $\tilde{\zeta}_{i j}^{(1)}$ from (5.21). Therefore we find the first element at the left hand side of (5.21)

$$
\begin{aligned}
& -\tilde{\zeta}_{1 j}^{(1)}+\delta_{1 j}=-\sum_{\substack{r \in R, R_{1} \\
r \neq 1}} \bar{A}_{1 r} \tilde{\zeta}_{r j}^{(1)}-2 \bar{A}_{0} \tilde{\zeta}_{i j}^{(1)}, \\
& r \neq 1: \bar{A}_{1 r}=\bar{A}_{1 r_{1}}=A_{1 r} ; \quad \bar{A}_{0}=A_{11} .
\end{aligned}
$$

Let us introduce matrices $M^{-1}$ and $\bar{M}^{-1}$. They are connected one with other as $\vec{A}$ and $\bar{A}^{-1}$

$$
\begin{aligned}
& M=(1-A)^{-1}=1+\left(F^{(2)}\right)^{-1} F^{(11)} ; \quad A=1-M^{-1} \\
& \left(\bar{M}^{-1}\right)_{1 r}=\left(\bar{M}^{-1}\right)_{1 r_{1}}=\left(M^{-1}\right)_{1 r} ;\left(\bar{M}^{-1}\right)_{0}=\left(\bar{M}^{-1}\right)_{i i} .
\end{aligned}
$$

Then the equation (5.23) for $\tilde{\zeta}_{i j}^{(1)}$ takes the form

$$
\sum_{r \in R_{1} R_{2}}\left\{\delta_{1 r}\left[1-2\left(\bar{M}^{-1}\right)_{0}\right]-\left(\bar{M}^{-1}\right)_{1 r}\right\} \zeta_{\tau j}^{(1)}=-\delta_{1 j}
$$

Taking into account (5.17) we write the equation for the reference $\stackrel{\bullet}{\mathrm{CF}} \hat{F}^{(2)}$

$$
\sum_{r \in R_{1} R_{2}}\left\{\delta_{1 r}\left[\frac{1}{F^{(2)}}-2 P_{0}\right]-P_{1 r}\right\} F_{r j}^{(2)}=-\delta_{1 j}
$$

Here the matrix $F$ is defined as

$$
\hat{P}=M^{-1}\left(F^{(2)}\right)^{-1} \quad \hat{P}^{-1}=F^{(2)}+F^{(11)} .
$$

Let us return to complex notations of bonds $i=\left(\mathbf{n}_{i}, \alpha_{i}\right)$ for relation (5.26). We shall express the hasic vectors of the Bravais lattice cell $\mathbf{a}_{1}, \mathbf{a}_{2}, \mathbf{a}_{3}$ in terms of vectors of the West's cell $e_{a}$

$$
\begin{aligned}
& \mathbf{a}_{1}=\frac{1}{2}\left[-\mathbf{e}_{1}+\mathbf{e}_{2}+\mathbf{e}_{3}\right] ; \mathbf{a}_{2}=\frac{1}{2}\left[\mathbf{e}_{1}+\mathbf{e}_{2}+\mathbf{e}_{3}\right] ; \mathbf{a}_{3}=\mathbf{e}_{2}, \\
& \mathbf{b}_{1}=2 \pi\left[-\mathbf{e}_{1}+\mathbf{e}_{3}\right] ; \mathbf{b}_{2}=2 \pi\left[\mathbf{e}_{1}+\mathbf{e}_{3}\right] ; \mathbf{b}_{3}=2 \pi\left[\mathbf{e}_{2}-\mathbf{e}_{3}\right] .
\end{aligned}
$$


Here $\mathbf{b}_{a}$ are the basic vectors of reciprocal lattice. Then after Fourier transformation over $\mathrm{n}$ the relation (5.26) takes the form

$$
\begin{aligned}
& \sum_{\alpha_{1}}\left\{\delta_{\alpha \alpha_{1}}\left[\frac{1}{1-m^{2}}-2 P_{0}\right]-P_{\alpha \alpha_{1}}(\mathbf{q})\right\} F_{\alpha_{1} \alpha^{\prime}}^{(2)}(\mathbf{q})=-\delta_{\alpha \alpha^{\prime}}, \\
& \left\{\left(1-m^{2}\right)^{-1}-\hat{P}(\mathbf{q})\right\} F^{(2)}(\mathbf{q})=-I .
\end{aligned}
$$

Here the matrix $P(\mathbf{q})$ has the form

$$
\begin{aligned}
& P(\mathbf{q})=\left(\begin{array}{cccc}
2 P_{0} & P_{3}(\mathbf{q}) & P_{1}(\mathbf{q}) & P_{2}(\mathbf{q}) \\
P_{3}(\mathbf{q}) & 2 P_{0} & P_{5}(\mathbf{q}) & P_{6}(\mathbf{q}) \\
P_{1}(\mathbf{q}) & P_{5}(\mathbf{q}) & 2 P_{0} & P_{4}(\mathbf{q}) \\
P_{2}(\mathbf{q}) & P_{6}(\mathbf{q}) & P_{4}(\mathbf{q}) & 2 P_{0}
\end{array}\right) \\
& P_{1}(\mathbf{q})=2 P_{2} \cos \frac{q_{x}}{2} ; P_{2}(\mathbf{q})=2 P_{1} \cos \frac{1}{4}\left(q_{x}-q_{y}+q_{z}\right) \\
& P_{3}(\mathbf{q})=2 P_{1} \cos \frac{1}{4}\left(q_{x}+q_{y}+q_{z}\right) ; P_{4}(\mathbf{q})=2 P_{1} \cos \frac{1}{4}\left(q_{x}+q_{y}-q_{z}\right) \\
& P_{5}(\mathbf{q})=2 P_{1} \cos \frac{1}{4}\left(q_{x}-q_{y}-q_{z}\right) ; P_{6}(\mathbf{q})=2 P_{2} \cos \frac{1}{4} q_{y}
\end{aligned}
$$

The quantities $P_{0}, P_{1}, P_{2}$ are expressed in the terms of functions $F^{(2)}, F_{1}$, $F_{2}$ with the aid of the relation

$$
\frac{1}{2} P(\mathbf{q}=0)=P=\left[F^{(2)}+F^{(11)}\right]^{-1} .
$$

Here $q_{x}, q_{y}, q_{z}$ are projection of dimensionless wave-vector $\mathbf{q}$ on the vectors $\mathbf{e}_{a}$ and in the first Brillouin zone $q_{a} \in[-\pi, \pi]$. From (5.32) we find

$$
\begin{aligned}
& P_{0}=\mathcal{D}^{-1} U_{0} ; P_{1}=\mathcal{D}^{-1} U_{1} ; P_{2}=\mathcal{D}^{-1} U_{2} \\
& P_{0}+2 P_{1}+P_{2}=\left(F^{(2)}+2 F_{1}+F_{2}\right)^{-1}=\frac{1}{8} \frac{Z_{R}}{c_{4}+b c_{2}-0.5 Z_{R} m^{2}} \\
& P_{0}-P_{2}=\left(F^{(2)}-F_{2}\right)^{-1}=\frac{1}{8} \frac{Z_{R}}{b c_{2}+a} \\
& \mathcal{D}=\frac{32}{Z_{R}}\left(b c_{2}+a\right)\left(b c_{2}+d\right)\left(c_{4}+b c_{2}-0.5 Z_{r} m^{2}\right) \\
& U_{0}=\left(3 b c_{2}+a+2 a b+2 d\right)+(4 b+3 d) b c_{2}+a d- \\
& -0.5 Z_{R} m^{2}\left(2 c_{4}+2 b c_{2}+d\right)-\frac{1}{8} Z_{R}^{2} m^{2} \\
& U_{1}=-\left(c_{4}-d\right)\left(b c_{2}+a\right)+0.5 Z_{R} m^{2}\left(b c_{2}+a\right) \\
& U_{2}=\left(-b c_{2}+a-2 d\right) c_{4}+(2 a-d) b c_{2}+a d- \\
& -0.5 Z_{R} m^{2}\left(-b c_{2}+a-2 d\right)-\frac{1}{8} Z_{R}^{2} m^{4} \\
& c_{4}=\cosh 4 \beta \tilde{\tilde{\zeta}} ; \quad c_{2}=\cosh 2 \beta \tilde{\tilde{\zeta}}
\end{aligned}
$$

Let us note that relations of (5.29) at $T>T_{c}$ were obtained firstly in [34]. The method developed in this work is acceptable only in the case $T>T_{c}$. Moreover in [34] the Sleter model corresponding to the Hamiltonian (5.1) 
at $\Phi=0$, was considered. For the mentioned above cases the matrix (5.30) coincides with corresponding matrix of [34].

The components of susceptibility tenzor (see next subsection ) will contain only certain eigenvalues of matrix $P(\mathbf{q})$. Therefore we shall consider the procedure of diagonalizing of equation (5.29). Let us introduce notation for eigenfunctions $U_{f \mu}(\mathbf{q})$ and eigenvalues $\epsilon_{\mu}(\mathbf{q})$ of matrix $\hat{P}(\mathbf{q})-2 P_{0} \hat{I}$. After unitary transformation we obtain

$$
\begin{aligned}
& \hat{\tilde{\mathcal{F}}}^{(2)}(\mathbf{q})=\hat{U}^{+}(\mathbf{q}) \hat{\mathcal{F}}^{(2)}(\mathbf{q}) \hat{U}(\mathbf{q}) ; \quad \hat{\mathcal{F}}^{(2)}(\mathbf{q})=\hat{U}(\mathbf{q}) \hat{\tilde{\mathcal{F}}}^{(2)}(\mathbf{q}) \hat{U}^{+}(\mathbf{q}) \\
& \mathcal{F}_{f f^{\prime}}^{(2)}(\mathbf{q})=\sum_{\mu} U_{f \mu}(\mathbf{q}) \tilde{\mathcal{F}}_{\mu}^{(2)}(\mathbf{q}) U_{f^{\prime} \mu}(\mathbf{q}) \\
& \tilde{\mathcal{F}}_{\mu}^{(2)}(\mathbf{q})=\left[\epsilon_{\mu}(\mathbf{q})+2 P_{0}-\left(1-m^{2}\right)^{-1}\right]^{-1}
\end{aligned}
$$

Here quantities $\epsilon_{\mu}(\mathbf{q})$ are to be found from the equation

$$
\begin{aligned}
& \epsilon^{4}-\left[\sum_{f=1}^{6} P_{f}^{2}(\mathbf{q})\right] \epsilon^{2}-2\left[P_{1}(\mathbf{q})\left(P_{2}(\mathbf{q}) P_{4}(\mathbf{q})+P_{3}(\mathbf{q}) P_{5}(\mathbf{q})\right)+\right. \\
& \left.+P_{6}(\mathbf{q})\left(P_{2}(\mathbf{q}) P_{3}(\mathbf{q})+P_{4}(\mathbf{q}) P_{5}(\mathbf{q})\right)\right] \epsilon+P_{1}^{2}(\mathbf{q}) P_{6}^{2}(\mathbf{q})+ \\
& +P_{2}^{2}(\mathbf{q}) P_{5}^{2}(\mathbf{q})+P_{3}^{2}(\mathbf{q}) P_{4}^{2}(\mathbf{q})-2 P_{2}(\mathbf{q}) P_{3}(\mathbf{q}) P_{4}(\mathbf{q}) P_{5}(\mathbf{q})- \\
& -2 P_{1}(\mathbf{q}) P_{6}(\mathbf{q})\left[P_{2}(\mathbf{q}) P_{5}(\mathbf{q})+P_{3}(\mathbf{q}) P_{4}(\mathbf{q})\right]=0
\end{aligned}
$$

We consider such values $\mathbf{q}$ for which the problem of eigenfunctions and eigenvalues due to the symmetry of $P(\mathbf{q})$ may lead to solution the equations of second order. For the values $\mathbf{q}=\left(q, q, q_{z}\right)$ we find

$$
\begin{aligned}
& 2 \epsilon_{1,2}(\mathbf{q})=P_{3}(\mathbf{q})+P_{4}(\mathbf{q}) \pm \sqrt{\mathcal{D}_{12}^{+}(\mathbf{q})} \\
& 2 \epsilon_{3,4}(\mathbf{q})=-P_{3}(\mathbf{q})-P_{4}(\mathbf{q}) \pm \sqrt{\mathcal{D}_{12}^{-}(\mathbf{q})}, \\
& \mathcal{D}_{12}^{ \pm}(\mathbf{q})=\left[P_{3}(\mathbf{q})-P_{4}(\mathbf{q})\right]^{2}+4\left[P_{1}(\mathbf{q}) \pm P_{2}(\mathbf{q})\right]^{2} ; \\
& U(\mathbf{q})=\left(\begin{array}{cccc}
\varphi_{11}^{+}(\mathbf{q}) & \varphi_{12}^{+}(\mathbf{q}) & \varphi_{11}^{-}(\mathbf{q}) & \varphi_{12}^{-}(\mathbf{q}) \\
\varphi_{11}^{+}(\mathbf{q}) & \varphi_{12}^{+}(\mathbf{q}) & -\varphi_{11}^{-}(\mathbf{q}) & -\varphi_{12}^{-}(\mathbf{q}) \\
\varphi_{12}^{+}(\mathbf{q}) & -\varphi_{11}^{+}(\mathbf{q}) & \varphi_{12}^{-}(\mathbf{q}) & -\varphi_{11}^{-}(\mathbf{q}) \\
\varphi_{12}^{+}(\mathbf{q}) & -\varphi_{11}^{+}(\mathbf{q}) & -\varphi_{12}^{-}(\mathbf{q}) & \varphi_{11}^{-}(\mathbf{q})
\end{array}\right) ; \\
& \varphi_{11}^{\delta}(\mathbf{q})=-\varphi_{22}^{\delta}(\mathbf{q})=\frac{1}{\sqrt{2}}\left[1+\delta r_{\delta}(\mathbf{q})\right]^{1 / 2} \text {, } \\
& \varphi_{12}^{\delta}(\mathbf{q})=\frac{\left[1-\delta r_{\delta}(\mathbf{q})\right]^{1 / 2}}{\sqrt{2}} \\
& r_{\delta}(\mathbf{q})=\frac{1}{2} R_{\delta}(\mathbf{q})\left[1+\frac{1}{4} R_{\delta}^{2}(\mathbf{q})\right]^{1 / 2} ; R_{\delta}(\mathbf{q})=\frac{P_{3}(\mathbf{q})-P_{4}(\mathbf{q})}{P_{1}(\mathbf{q})+\delta P_{2}(\mathbf{q})} .
\end{aligned}
$$

For the values $\mathbf{q}=\left(q_{x}, q_{y}, 0\right)$ we obtain

$$
2 \epsilon_{1,3}(\mathbf{q})=P_{1}(\mathbf{q})+P_{6}(\mathbf{q}) \pm \sqrt{\mathcal{D}_{32}^{+}(\mathbf{q})}
$$




$$
\begin{aligned}
& 2 \epsilon_{2,4}(\mathbf{q})=-P_{1}(\mathbf{q})-P_{6}(\mathbf{q}) \pm \sqrt{\mathcal{D}_{32}^{-}(\mathbf{q})} \\
& \mathcal{D}_{32}^{ \pm}(\mathbf{q})=\left[P_{1}(\mathbf{q})-P_{6}(\mathbf{q})\right]^{2}+4\left[P_{3}(\mathbf{q}) \pm P_{2}(\mathbf{q})\right]^{2} ; \\
& U(\mathbf{q})=\left(\begin{array}{cccc}
\varphi_{11}^{+}(\mathbf{q}) & \varphi_{12}^{+}(\mathbf{q}) & \varphi_{11}^{-}(\mathbf{q}) & \varphi_{12}^{-}(\mathbf{q}) \\
\varphi_{12}^{+}(\mathbf{q}) & \varphi_{11}^{+}(\mathbf{q}) & \varphi_{12}^{-}(\mathbf{q}) & -\varphi_{11}^{-}(\mathbf{q}) \\
\varphi_{11}^{+}(\mathbf{q}) & \varphi_{12}^{+}(\mathbf{q}) & -\varphi_{11}^{-}(\mathbf{q}) & -\varphi_{12}^{-}(\mathbf{q}) \\
\varphi_{12}^{+}(\mathbf{q}) & -\varphi_{11}^{+}(\mathbf{q}) & -\varphi_{12}^{-}(\mathbf{q}) & \varphi_{11}^{-}(\mathbf{q})
\end{array}\right), \\
& \varphi_{11}^{\delta}(\mathbf{q})=-\varphi_{22}^{\delta}(\mathbf{q})=\frac{\left[1+\delta r_{\delta}(\mathbf{q})\right]^{1 / 2}}{\sqrt{2}} \\
& \varphi_{12}^{\delta}(\mathbf{q})=\frac{\left[1-\delta r_{\delta}(\mathbf{q})\right]^{1 / 2}}{\sqrt{2}}, \\
& r_{\delta}(\mathbf{q})=\frac{R_{\delta}(\mathbf{q})}{2}\left[1+\frac{1}{4} R_{\delta}^{2}(\mathbf{q})\right]^{1 / 2} ; R_{\delta}(\mathbf{q})=\frac{P_{1}(\mathbf{q})-P_{6}(\mathbf{q})}{P_{3}(\mathbf{q})+\delta P_{2}(\mathbf{q})} .
\end{aligned}
$$

Let us note that in [34] the eigenvalues were obtained only for vector $\mathbf{q}=\left(q, q, q_{z}\right)$ and the long-range interaction was not considered.

\subsection{Susceptibility of $\mathrm{KD}_{2} \mathrm{PO}_{4}$ system}

The tensor of isotermical susceptibility of $\mathrm{K}_{2} \mathrm{PO}_{4}$ system can be expressed in terms of the pair $\mathrm{CF}^{L} \mathcal{F}_{\alpha \beta}^{(2)}(\mathbf{q})$ taking into consideration long-range interaction in MFA in the following way

$$
\begin{aligned}
& \chi_{a b}(\mathbf{q})=\sum_{\alpha, \beta} d_{\alpha a} d_{\beta b}{ }^{L} \mathcal{F}_{\alpha \beta}^{(2)}(\mathbf{q}), \\
& { }^{L} \hat{\mathcal{F}}^{(2)}(\mathbf{q})=\left\{\left[\hat{\mathcal{F}}^{(2)}(\mathbf{q})\right]^{-1}-\beta \hat{J}(\mathbf{q})\right\}^{-1}, \\
& d_{\alpha z}=d_{c} ; \quad d_{x}=d_{1 x}=-d_{3 x} ; \quad d_{2 x}=d_{4 x}=0, \\
& d_{y}=d_{2 y}=-d_{4 y} ; \quad d_{1 y}=-d_{3 y}=0 .
\end{aligned}
$$

Here $d_{\alpha a}$ is an effective dipolar moment of hydrogen bond. It contains the contribution of ionic subsystem via spin-phonon interaction. We write the longitiudinal and transversal components of tenzor $\chi_{a b}(q)$ in terms of eigenfunctions $W_{\alpha \mu}(\mathbf{q})$ and eigenvalues $\tilde{\mathcal{F}}_{\mu}^{(2)}(\mathbf{q})$ of the matrix ${ }^{L_{\mathcal{F}}}{ }_{\alpha \beta}^{(2)}(\mathbf{q})$.

$$
\begin{aligned}
& \chi_{z z}(\mathbf{q})=d_{c}^{2} \sum_{\mu} \tilde{\mathcal{F}}_{\mu}^{(2)}(\mathbf{q}) f_{\mu}^{2}(\mathbf{q}) ; f_{\mu}(\mathbf{q})=\sum_{f} W_{f \mu}(\mathbf{q}), \\
& \chi_{x x}(\mathbf{q})=d_{x}^{2} \sum_{\mu} \tilde{\mathcal{F}}_{\mu}^{(2)}(\mathbf{q})\left[W_{1 \mu}(\mathbf{q})-W_{3 \mu}(\mathbf{q})\right]^{2} \\
& \chi_{y y}(\mathbf{q})=d_{y}^{2} \sum_{\mu} \tilde{\mathcal{F}}_{\mu}^{(2)}(\mathbf{q})\left[W_{2 \mu}(\mathbf{q})-W_{4 \mu}(\mathbf{q})\right]^{2}
\end{aligned}
$$

The symmetry of matrix $\hat{\mathcal{F}}^{(2)}(\mathbf{q})$ which is related with the symmetry of matrix $\hat{P}(\mathbf{q})$ at arbitrary value of $\mathbf{q}$ does not coincide with the symmetry of matrix $J_{\alpha \beta}(\mathbf{q})$. Here we consider only such values of $\mathbf{q}$ when $P(\mathbf{q})$ and 
$I(\mathbf{q})$ has equal eigenfunctions. Then on the basis (5.38a) and (5.34) one can write

$$
\begin{aligned}
& \tilde{\mathcal{F}}_{\mu}^{(2)}(\mathbf{q})=\left[-\left(1-m^{2}\right)^{-1}+2 P_{0}+\epsilon_{\mu}(\mathbf{q})-\nu_{\mu}(\mathbf{q})\right]^{-1}, \\
& \epsilon_{\mu}(\mathbf{q})=\sum_{\alpha, \alpha^{\prime}} W_{\alpha \mu}(\mathbf{q}) P_{\alpha \alpha^{\prime}}(\mathbf{q}) W_{\alpha^{\prime} \mu}(\mathbf{q})-2 P_{0}, \\
& \nu_{\mu}(\mathbf{q})=\sum_{\alpha, \alpha^{\prime}} W_{\alpha \mu}(\mathbf{q}) J_{\alpha \alpha^{\prime}}(\mathbf{q}) W_{\alpha^{\prime} \mu}(\mathbf{q}) .
\end{aligned}
$$

We present here several cases of application of relation (5.40). At $\mathbf{q} \rightarrow 0$ we use the expressions for $J_{\alpha \beta}(\mathbf{q})$ from [59]

1. $\mathbf{q}=\hat{q}_{z}=\left(0,0, q_{z}\right)$

$$
\begin{aligned}
& \hat{J}\left(\hat{q}_{z}\right)=\left(\begin{array}{llll}
J_{11}\left(\hat{q}_{z}\right) & J_{12}\left(\hat{q}_{z}\right) & J_{13}\left(\hat{q}_{z}\right) & J_{12}\left(\hat{q}_{z}\right) \\
J_{12}\left(\hat{q}_{z}\right) & J_{11}\left(\hat{q}_{z}\right) & J_{12}\left(\hat{q}_{z}\right) & J_{13}\left(\hat{q}_{z}\right) \\
J_{13}\left(\hat{q}_{z}\right) & J_{12}\left(\hat{q}_{z}\right) & J_{11}\left(\hat{q}_{z}\right) & J_{12}\left(\hat{q}_{z}\right) \\
J_{12}\left(\hat{q}_{z}\right) & J_{13}\left(\hat{q}_{z}\right) & J_{12}\left(\hat{q}_{z}\right) & J_{11}\left(\hat{q}_{z}\right)
\end{array}\right), \\
& J_{11}\left(\hat{q}_{z}\right) \stackrel{\hat{q}_{z} \rightarrow 0}{\longrightarrow} J_{11}+a_{z} ; \quad J_{12}\left(\hat{q}_{z}\right) \stackrel{q_{z} \rightarrow 0}{\longrightarrow} J_{12}, \\
& J_{13}\left(\hat{q}_{z}\right) \stackrel{q_{z} \rightarrow 0}{\longrightarrow} J_{13}-a_{z} \\
& \hat{W}\left(\hat{q}_{z}\right) \equiv \hat{U}\left(\hat{q}_{z}\right)=\frac{1}{2}\left(\begin{array}{cccc}
1 & 1 & 1 & 1 \\
1 & 1 & -1 & -1 \\
1 & -1 & 1 & -1 \\
1 & -1 & -1 & 1
\end{array}\right) \text {, } \\
& \nu_{1,3}\left(\hat{q}_{z}\right)=J_{11}\left(\hat{q}_{z}\right)+J_{13}\left(\hat{q}_{z}\right) \pm 2 J_{12}\left(\hat{q}_{z}\right) \stackrel{q_{z \rightarrow 0}}{\longrightarrow} J_{11}+J_{13} \pm 2 J_{12}, \\
& \nu_{2,4}\left(\hat{q}_{z}\right)=J_{11}\left(\hat{q}_{z}\right)-J_{13}\left(\hat{q}_{z}\right) \stackrel{q_{z} \rightarrow 0}{\longrightarrow} J_{11}-J_{13} \pm 2 a_{z}, \\
& \epsilon_{1,2}\left(\hat{q}_{z}\right)=2\left[P_{2} \pm P_{1} \cos \frac{q_{z}}{4}\right] ; \epsilon_{3,4}\left(\hat{q}_{z}\right)=-2 P_{2} \text {, } \\
& \frac{\chi_{z z}\left(\hat{q}_{z}\right)}{\left(2 d_{z}\right)^{2}}=\left\{2\left(P_{0}+2 P_{1}+P_{2}\right)-8 P_{1} \sin ^{2} \frac{q_{z}}{8}-\left(1-m^{2}\right)^{-1}-\nu_{1}\left(q_{z}\right)\right\}^{-1} \text {, } \\
& \frac{\chi_{a a}\left(\hat{q}_{x}\right)}{2\left(2 d_{a}\right)^{2}}=\left\{2\left(P_{0}-P_{2}\right)-\left(1-m^{2}\right)^{-1}-\nu_{2}\left(q_{z}\right)\right\}^{-1} ; a=x, y \text {. }
\end{aligned}
$$

2. $\mathbf{q}=\hat{q}=(\epsilon, \epsilon, 0), \epsilon \rightarrow 0$

$$
\begin{aligned}
& J(\hat{q})=\left(\begin{array}{llll}
J_{11}(\hat{q}) & J_{12}(\hat{q}) & J_{13}(\hat{q}) & J_{14}(\hat{q}) \\
J_{12}(\hat{q}) & J_{11}(\hat{q}) & J_{14}(\hat{q}) & J_{13}(\hat{q}) \\
J_{13}(\hat{q}) & J_{14}(\hat{q}) & J_{11}(\hat{q}) & J_{12}(\hat{q}) \\
J_{14}(\hat{q}) & J_{13}(\hat{q}) & J_{12}(\hat{q}) & J_{11}(\hat{q})
\end{array}\right) \\
& J_{11}(\hat{q})=J_{11}+a_{x y} ; \quad J_{12}(\hat{q})=J_{12}+a_{x y} \\
& J_{14}(\hat{q})=J_{12}-a_{x y} ; \quad J_{13}(\hat{q})=J_{13}-a_{x y} \\
& \hat{W}(\hat{q})=\hat{U}(\hat{q})=\frac{1}{2}\left(\begin{array}{cccc}
1 & 1 & 1 & 1 \\
1 & 1 & -1 & -1 \\
1 & -1 & 1 & -1 \\
1 & -1 & -1 & 1
\end{array}\right) \\
& \nu_{1,3}(\hat{q})=J_{11}(\hat{q})+J_{13}(\hat{q}) \pm\left[J_{12}(\hat{q})+J_{14}(\hat{q})\right]=J_{11}+J_{13} \pm 2 J_{12}
\end{aligned}
$$




$$
\begin{aligned}
& \nu_{2,4}(\hat{q})=J_{11}(\hat{q})-J_{13}(\hat{q}) \pm\left[J_{12}(\hat{q})-J_{14}(\hat{q})\right] \\
& \nu_{2}(\hat{q})=J_{11}-J_{13}+4 a_{x y} ; \quad \nu_{4}(\hat{q})=J_{11}-J_{13} \\
& \epsilon_{1,3}(\hat{q})= \pm 4 P_{1}+2 P_{2} ; \quad \epsilon_{2,4}(\hat{q})=-2 P_{2} \\
& \frac{\chi_{z z}(\hat{q})}{\left(2 d_{z}\right)^{2}}=\left\{2\left(P_{0}+2 P_{1}+P_{2}\right)-\left(1-m^{2}\right)^{-1}-\nu_{1}(\hat{q})\right\}^{-1} \\
& \frac{\chi_{a a}(\hat{q})}{\left(2 d_{a}\right)^{2}}=\sum_{\mu=2,4}\left\{2\left(P_{0}-P_{2}\right)-\left(1-m^{2}\right)^{-1}-\nu_{\mu}(\hat{q})\right\}^{-1}
\end{aligned}
$$

3. $\mathbf{q}=\hat{a}, \hat{x}=(\epsilon, 0,0), \hat{y}=(0, \epsilon, 0), \epsilon \rightarrow 0$

$$
\begin{aligned}
& J(\hat{a})=\left(\begin{array}{llll}
J_{11}(\hat{a}) & J_{12}(\hat{a}) & J_{13}(\hat{a}) & J_{12}(\hat{a}) \\
J_{12}(\hat{a}) & J_{22}(\hat{a}) & J_{12}(\hat{a}) & J_{24}(\hat{a}) \\
J_{13}(\hat{a}) & J_{12}(\hat{a}) & J_{11}(\hat{a}) & J_{12}(\hat{a}) \\
J_{12}(\hat{a}) & J_{24}(\hat{a}) & J_{12}(\hat{a}) & J_{22}(\hat{a})
\end{array}\right), \\
& J_{11}(\hat{a})=J_{11}+b(\hat{a}) ; \quad J_{22}(\hat{a})=J_{11}+a(\hat{a}), \\
& J_{13}(\hat{a})=J_{13}-b(\hat{a}) ; \quad J_{24}(\hat{a})=J_{13}-a(\hat{a}), \\
& J_{12}(\hat{a})=J_{12} ; \quad b(\hat{a})=a(\hat{a}), \\
& \hat{W}(\hat{a})=\hat{U}(\hat{a})=\frac{1}{2}\left(\begin{array}{cccc}
1 & 1 & \sqrt{2} & 0 \\
1 & -1 & 0 & \sqrt{2} \\
1 & 1 & -\sqrt{2} & 0 \\
1 & -1 & 0 & -\sqrt{2}
\end{array}\right) \text {, } \\
& \nu_{1,3}(\hat{q})=J_{11}(\hat{a})+J_{13}(\hat{a}) \pm J_{12}(\hat{a})=J_{11}+J_{13} \pm 2 J_{12} \text {, } \\
& \nu_{2}(\hat{q})=J_{11}(\hat{a})-J_{13}(\hat{a})=J_{11}-J_{13}+2 b(\hat{a}), \\
& \nu_{4}(\hat{q})=J_{22}(\hat{a})-J_{24}(\hat{a})=J_{11}-J_{13}+2 a(\hat{a}) \text {, } \\
& \epsilon_{1,2}(\hat{q})= \pm 4 P_{1}+2 P_{2} ; \quad \epsilon_{3,4}(\hat{q})=-2 P_{2} \text {, } \\
& \frac{\chi_{z z}(\hat{q})}{2\left(2 d_{z}\right)^{2}}=\left\{2\left(P_{0}+2 P_{1}+P_{2}\right)-\left(1-m^{2}\right)^{-1}-\nu_{1}(\hat{q})\right\}^{-1} \text {, } \\
& \frac{\chi_{a a}(\hat{q})}{2\left(2 d_{a}\right)^{2}}=\left\{2\left(P_{0}-P_{2}\right)-\left(1-m^{2}\right)^{-1}-\nu_{\mu}(\hat{q})\right\}^{-1} ; \mu=\left\{\begin{array}{ll}
2, & a=x \\
4, & a=y
\end{array}\right. \text {. }
\end{aligned}
$$

More detailed investigation of $\vec{q}$-dependence for susceptibility of $\mathrm{KD}_{2} \mathrm{PO}_{4}$ system will be carried out in the separate paper.

\section{Conclusion}

Thus in the present work we have suggested the cluster method for computation of the correlation functions of the Ising-type systems with short-range interactions and with arbitrary value of spin. The method is based on the calculation of generating function (that is the logarithm of partition function for a system in nonuniform external field). Here we restricted ourselves only to the first order of cluster approximation for generating function. The investigation of some models $\left(S^{z}= \pm 1, S^{z}=-2,0,+2\right)$ has been carried out within two-particle cluster approximation. The static susceptibility of $\mathrm{K} \mathrm{D}_{2} \mathrm{PO}_{4}$ crystal has been obtained within four-particle cluster approximation. 
The obtained here form of equations for CFs is similar to the form which one can obtain within random phase approximation (molecular field approximation for generating function). Moreover in the case of Bravais lattice the q-dependence of CFs is similar to one obtained within random-phase approximation. Nevertheless within cluster approximation we obtain the more precise expressions for the temperature depending coefficients. In the case of tree-like lattices, in particular, for $1 \mathrm{D}$ lattices, the cluster approximation gives the exact expression for all static characteristics of the Ising-type systems. The more detailed study including numerical calculations of expressions obtained in the present work will be given elsewhere.

\section{References}

[1] Nagaev E.L. Magnets with complicated exchange interactions. - M.: Nauka, 1988. - 232p. (in Russian).

[2] Mitsek A.I. Phase transitions in the crystals with magnetic structure. - Kiev: Naukova dumka, 1989. - 320p. (in Russian).

[3] Steiner M., Villain J., Windsor C.G. Theoretical and experimental studies of one-dimensional magnetic systems // Adv. Phys. - 1976. - 25, No 2. P.87-209.

[4] Vaks V.G. Introduction to the microscopic theory of ferroelectrics. - M.: Nauka, 1973. - 327p. (in Russian).

[5] Blinc R., Zeks B. Soft modes in ferroelectrics and antiferroelectrics. Amsterdam: North-Holland Publishing Company; New York: American Elsevier Publishing Company, 1974.

[6] Khachaturyan A.G. Phase transition theory and stucture of solid solutions. M.: Nauka, 1974. - 384p. (in Russian).

[7] Yukhnovskii I.R., Gurskii Z.A. Quantum-statistical theory of disordered systems. - Kiev: Naukova dumka, 1991. - 228p. (in Russian).

[8] Shulepov Yu.V., Aksenenko E.V. Lattice gas. - Kiev: Naukova dumka, 1981. - 268p. (in Russian).

[9] Yukhnovskii I.R., Levitskii R.R., Sorokov S.I. The theory of quasisipin system taking into account short-range interaction as a reference system. Expansion over inverse radius of long-range interaction. - Kiev, 1986. - 48p. -(Preprint/AN UkrSSR. Inst. Teor. Fiz., ITF-86-132R). (in Russian).

[10] Yukhnovskii I.R., Levitskii R.R., Sorokov S.I. The theory of quasispin system taking into account short-range interaction as a reference system. Summation of reducible diagrams. - Kiev, 1986. -48p. -(Preprint/AN ÚkrSSR. Inst. Teor. Fiz., ITF-86-154R). (in Russian).

[11] Yukhnovskii I.R., Levitskii R.R., Sorokov S.I., Derzhko O.V. Theory of quasispin system described by quasi-one-dimensional Ising model in the transversal field with basic account of short-range interactions. //Izv. AN SSSR, ser.fiz. - 1991. - 55, No 3. - P.481-490. (in Russian).

[12] Levitskii R.R., Sorokov S.I. On the theory of quasi-spin system in the basic approach. Consideration of Ising fluctuations. - Lvov, 1992.-36p. -(Preprint/AN Ukrainy, Inst. Fiz. Kond. Sistem, IFKS-92-14R). (in Russian).

[13] Kubo R. Generalized cumulant expansion method//J. Phys. Soc. Japan. 1962. - 17, No 7. - P.1100-1120.

[14] Smart J.S. Effective field theories of magnetism. - Philadelphia-London: W.B.Saunders company, 1966.

[15] Strieb B., Callen H.B. Cluster expansion for Heisenberg ferromagnet//Phys. Rev. - 1963. - 130, No 5. - P.1798-1808.

[16] Blinc R., Svetina S. Cluster approximation for order-disorder type hydrogenbounded ferroelectrics. I. Small cluster//Phys. Rev. - 1966. - 147, No 2. P.423-429. 
[17] Blinc R., Svetina S. Cluster approximation for order-disorder-type hydrogenbounded ferroelectrics. II. Application to $\mathrm{KH}_{2} \mathrm{PO}_{4} / /$ Phys. Rev. - 1966. 147, No 2. - P.430-438.

[18] Vaks V.G., Zinenko V.I. On the theory of ferroelectrics of $\mathrm{KH}_{2} \mathrm{PO}_{4}$-type. //Zh.Eksp. i Teor.Fiz. - 1983. -64, No 2. -P.650-664.(in Russian).

[19] Levitskii R.R., Korinevskii N.A., Stasyuk I.V. Theory of proton ordering in ferro- and antiferroelectrics of ortophosphate type.//Ukr.Fiz.Zh. - 1974. -19, No 8 - P.1289-1297. (In Russian).

[20] Vaks V.G., Zein N.E., Strukov B.A. On the theory of ferroelectric of $\mathrm{K} \mathrm{H}_{2} \mathrm{PO}_{4}$ - KDP-type//Phys. Stat. Sol.(a). - 1975. - 30, No 2. - P.801-819.

[21] Vaks V.G., Zinenko V.I., Shneider V.E. Microscopic theories of the structural phase transitions of order-disorder type in crystals. //Usp.Fiz.Nauk. -1983.141, No 4 -P.626-673.(in Russian).

[22] Schmidt V.H. Review of order-disorder models for KDP-family crystals//Ferroelectrics. - 1987. - 72. - P.157-173.

[23] Blinc R., Zeks B. Proton order-disorder in $\mathrm{K} \mathrm{H}_{2} \mathrm{PO}_{4}$-type ferroelectrics. Slater theory and Ising model in a transverse tunneling field//Ferroelectrics. - 1987.
- 72. - P.193-227.

[24] Vaks V.G., Zein N.E. On the theory of phase transitions in solid solutions //Zh.Eksp.i Teor.Fiz. - 1974. - 67, No 9. - P.1082-1100. (in Russian). [25] Kikuchi R. A theory of cooperative phenomena//Phys. Rev. - 1951. - 81,
No 6. - P.988-1003.

[26] Morita T. General structure of the dictribution function for the Heisenberg model and the Ising model//J. Math. Phys. - 1972. - 13, No 1. - P.115-123.

[27] Tanaka T., Libelo L.F. Effects of nearest-neghbor four-spin correlation upon the critical properties of the spin - $1 / 2$ Heisenberg ferromagnet//Phys. Rev. B. $-1975 .-12$, No 5. - P.1790-1795.

[28] Aggarwal S.K., Tanaka T. Application of the cluster variation method to the fcc Ising ferromagnet//Phys. Rev. B. - 1977. - 16, No 9. - P.3963-3990. [29] Bell J.M. Modified form of the cluster-variation method//Phys. Rev. B. -
1987. - 35, No 8. - P.3783-3789.

[30] Sanchez J.M., Ducastelle F., Gratias D. Generalized cluster description of multicomponent systems/!"'ysica. - 1984. - 128A, No 1-2. - P.334-350.

[31] Zubkus V.E.,Lupinskas S. Modified cluster-field method for statistics of ordering systems//J. Phys. Condens. Matter. - 1990. - 2. - P.1753-1763.

[32] Levitskii R.R., Korinevsky N.A., Stasyuk I.V. Distribution function an thermodynamic properties of $\mathrm{KD}_{2} \mathrm{PO}_{4}$ and $\mathrm{ND}_{4} \mathrm{D}_{2} \mathrm{PO}_{4}$ type ferroelectrics//Phys. Stat. Sol.(b). - 1978, -88, No 1. - P.51-63.

[33] Levitskii R.R., Zachek I.R., Varanitskii V.N. Distribution functions and relaxation processes of the Ising model. Cluster approximation. - Kiev, 1978. -40 p. (Preprint/AN UkrSSR. Inst. Teor. Fiz.; ITF-78-41P). (In Russian).

[34] Havlin S., Sompolinsky H. Cluster approximation for q-dependent correlations in magnetic and ferroelectric system// Phys. Rev. B. - 1982. - 25, No 9,
P.5828-5835.

[35] Yukhnovskii I.R., Levitskii R.R., Sorokov S.I. Thermodynamics and distribution functions for the Ising model. Two-particle cluster approximation. Kiev, 1986. - 33p. - (Preprint/AN UkrSSR. Inst.Teor.Fiz.; ITF-86-142p). (in Russian).

[36] Sorokov S.I., Levitskii R.R., Baran O.R. The study of Ising model within cluster expansion method. - Lviv, 1992. - 47p. - (Preprint/AN Ukrainy. Inst. Fiz. Kond. System; IFKS-92-18U). (in Ukrainian). [37] Sorokov S.I., Levitskii R.R., Baran O.R. Two-particle cluster approximation
for Ising-type model with arbitrary value of spin. 1. The Blume-Capel-Griffiths model. - Lviv,1993. - 47p. - (Preprint/AN Ukrainy. Inst.Fiz.Kond.System; 1F KS-93-1U). (in Ukrainian).

[38] Stasyuk I.V., Levitskii R.R., Korinevskii N.A. Dynamics of protons in compounds of KDP type within cluster approximation. - Kiev, 1975.-24p. (Preprint/AN UkrSSR. Inst. Teor. Fiz.; ITF-75-121R). (In Russian). 
[39] Stasyuk I.V., Levitskii R.R., Korinevsky N.A. Collective vibrations of protons in compounds of $\mathrm{KH}_{2} \mathrm{PO}_{4}$-type. The cluster approximation//Phys. Stat. Sol. (b). - 1979. - 91, No 2. - P.541-550.

[40] Korinevskii N.A., Levitskii R.R. Dynamical theory of ortophosphate within cluster approximation //Teor.Mat.Fiz. -1980. -42, No 3.-P.416-429. (in Russian).

[41] Levitskii R.R., Stasyuk I.V., Korinevsky N.A. Dynamics of ferroactive crystals of orthophosphate type //Ferroelectrics. - 1978. - 21. - P.481-483.

[42] Levitskii R.R., Sorokov S.I. The theory of quasispin system taking into account short-range interaction as a reference system within two-particle cluster approximation. Application to the Heisenberg model. - Kiev, 1987.-44p. (Preprint/AN UkrSSR. Inst. Teor. Fiz.; ITF-87-28R). (in Russian). [43] Levitskii R.R., Sorokov S.I. Dynamics and thermodynamic properties of the
Ising model in a transverse field. The two-particle cluster approximation. Kiev, 1988. - 48p. - (Preprint/AN UkrSSR. Ints. Teor. Fiz.; ITF-88-34R). (in Russian).

[44] Statistical Physics of Phase transition and Superfluidity, ed. M.Chretien et.al. Gordon and Breach Science Publishers, Vol.1,2, New York, 1968.

[45] Baxter R. Exactly solved models in statistical mechanics. -London New York Paris San Diego San Francisko Sao Paulo Sydney Tokyo Toronto; Academic

[46] Suzuki M., Kubo R. Dynamics of the Ising model near the Curie temperature// J.Phys.Soc.Japan. - 1968. -24, No 1.-P.51-61.

[47] Zhelifonov M.P. The highest correlation functions of the Ising type ferromagnet. The case of linier model for spin $S=1 / 2$. -Teor.Mat.Fiz. $-1971,8$, No 3 , P.401-411. (in Russian).

[48] Kessel A.R., Berim G.O. Magnitnyi rezonans izingovskikh magnetikov. - M.: Mir, 1982. - 147p. (in Russian).

[49] Berim G.O., Kessel A.R. Kinetics of the Ising magnet. 1. General theory //Physica. -1980, -102A, No 1. -P.112-126.

[50] Berim G.O., Kessel A.R. Kinetics of the Ising magnet. II. One-dimensional model //Physica. -1980. -102A, No 1. -P.127-144.

[51] Levitski R.R., Zachek I.R., Tokarchuk M.V. On the theory of relaxational fenomena in quasi-one-dimensional ferroelectrics of order-disorder type. Lvov, 1991. -40p. - (Preprint/AN UkrSSR. Inst. Fiz. Cond. Sistem; IFKS91-65R). (in Russian).

[52] Glauber R.J. Time-dependent statistics of the Ising model //J. Math. Phys.
-1963. -4, No 2. -P.294-307.

[53] Mamada H., Takano F. New approximate method for Ising system //J. Phys.

[54] Huang H.W. First-order approximation for the time-dependent Ising model //Phys. Rev. B. -1975. -11, No 3. -P.1144-1151.

[55] Vlasova A.A., Shneider V.E. Relaxational dynamics of the Ising model within cluster approximation //Zh. Eksp. i Teor. Fiz. -1977.-73, No 10.-P.1493-1498. (in Russian).

[56] Levitskii R.R., Zachek I.R., Varanitskii V.I. Relaxational processes described by the Ising model. Cluster approximation //Ukr. Fiz. Zh. -1979. -24, No 10.
-P.1486-1495.

[57] Blume M., Emery V.J., Griffiths R.B. Ising model for the $\lambda$ transition and phase separation in $\mathrm{He}^{3}-\mathrm{He}^{4}$ mixtures //Phys. Rev. A. -1971. -4, No 3 .
- 1071-1077.

[58] Levitskii R.R., Sorokov S.I., Sokolovskii R.O. Thermodynamics and relaxational dynamics of disiordered Ising model. Two-site cluster approximation. -Lviv, 1993. -43p. -(Preprint Ukr. Acad. Sci; Inst. Fiz. Cond Sistem, IFKS93-17U), (in Ukrainian).

[59] Stasyuk I.V., Kaminskaya N.M. Theory of spontaneous polarization and deformation of $\mathrm{K}_{2} \mathrm{PO}_{4}$ type ferroelectrics. II. Consideration of peculiarities in interproton interaction //Ukr. Fiz. Zh. -1974. -19, No 2.-P.244-252. 